\title{
Éléments de microscopie
}

\author{
G. Roblin \\ Institut d'Optique, B.P. 147, 91403 Orsay cedex, France
}

\begin{abstract}
Résumé : Après un bref exposé sur les caractéristiques essentielles de la microscopie et les propriétés du microscope sont abordés deux chapitres particuliers du domaine de cette optique instnumentale. L'observation des objets de phase plus généralement rencontrés en microscopie, non visibles naturellement, grâce aux techniques de contraste de phase et d'interférométrie est tout d'abord présentée. La récente introduction des méthodes d'obtention d'images par microscopie optique à balayage, notamment par le procédé confocal, et ses possibilités sont le second sujet traité.
\end{abstract}

\section{INTRODUCTION À LA MICROSCOPIE}

\subsection{Le microscope simplifié}

Le microscope simplifié (fig. 1) n'est constitué que de l'objectif $O b$ et de l'oculaire $O c$, chacun d'eux assimilè à une lentille mince convergente, et de deux diaphragmes $P_{o}^{\prime}$ et $D_{c}^{\prime}$ respectivement pupille de sortie de l'objectif et diaphragme de champ de l'oculaire tous deux placés dans l'espace intermédiaire (espace situé entre objectif et oculaire).

L'objectif doit donner de l'objet une image réelle agrandie devant être examinée par l'oculaire jouant le rôle de loupe. Pour que cet agrandissement soit suffisamment appréciable l'objet $A B$ doit être placé très près en avant du foyer objet $F_{o b}$ de l'objectif. Néanmoins pour limiter l'encombrement de l'instrument tant pour des raisons de stabilité que d'ergonomie (l'observateur doit placer l'œil derrière l'oculaire et pouvoir déplacer manuellement l'objet sur la platine), ce grandissement satisfaisant nécessite de l'objectif que sa distance focale soit la plus courte possible. L'objet très proche de son foyer objet sera donc pratiquement à une distance de la lentille égale à sa distance focale et, dans le cas réel, très proche de la face avant de l'objectif. La distance de l'objet au premier dioptre de l'objectif ou distance frontale sera très faible.

Dans le plan de l'image intermédiaire $A^{\prime} B^{\prime}$. image réelle renversée donnée par l'objectif est toujours un diaphragme (ou l'image d'un diaphragme réel situé dans un autre espace). $\mathrm{Ce}$ diaphragme de champ $D_{c}^{\prime}$, diaphragme vu du centre de la pupille $P_{o}^{\prime}$ sous l'angle le plus faible, limite toujours le champ de pleine lumière. Il a aussi pour effet de supprimer la lumière 
parasite engendrée par la lumière diffusée à partir des points éclairés de l'objet en dehors de ses limites. Cette image intermédiaire est toujours par construction (de conception fondamentale) dans le plan focal objet $F_{o c}$ de l'oculaire. L'image définitive $A$ " $B$ " donnée par l'oculaire est donc à l'infini. Ainsi le plan objet du microscope $F_{m}$ est son plan focal objet.

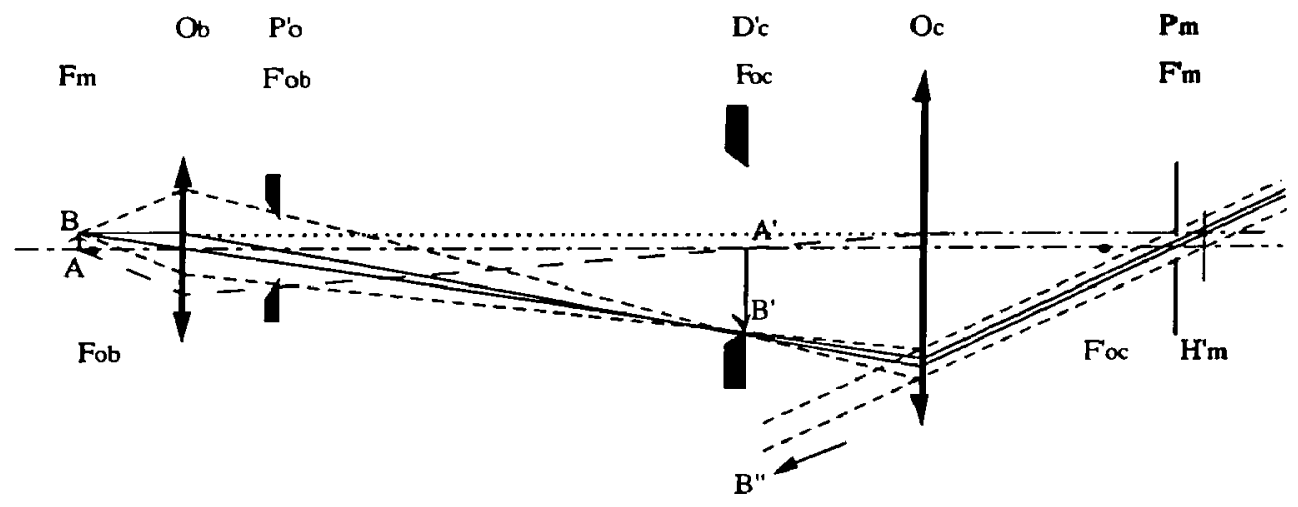

Figure 1 : Schéma du microscope simplifié

Tous les rayons issus de l'objectif contribuant au champ de pleine lumière limité par le diaphragme de champ passent par la pupille de l'objectif $P_{o}^{\prime}$, diaphragme vu du centre du champ sous l'angle le plus faible, matérialisée par un diaphragme (ou une image de diaphragme) placé par construction dans le plan focal image de l'objectif, la pupille d'entrée d'un microscope étant toujours par définition située à l'infini. Ainsi le plan focal image du microscope $F_{m}^{\prime}$ est confondu avec le plan de sa pupille de sortie $P_{m}^{\prime}$. Cette pupille est l'anneau oculaire du microscope où l'observateur devra placer sa propre pupille d'œil.

\subsection{Ouverture numérique et limite de résolution}

\subsubsection{L'ouverture mumérique}

Considérons un objectif de microscope observant un point $A$ d'un objet d'indice $\mathrm{n}_{0}$, recouvert d'une lamelle d'indice $\mathrm{n}_{\mathrm{V}}$, séparée de la lentille frontale de l'objectif par un liquide d'immersion d'indice n. Supposons que le rayon ABCD (fig. 2) soit le plus incliné sur l'axe optique capable d'entrer dans l'objectif et d'en ressortir. Son inclinaison à la traversée des dioptres plans séparant les milieux prend les valeurs successives $u_{0}, u_{V}, U$ et

$$
\mathbf{n}_{0} \sin \mathbf{u}_{0}=\mathbf{n}_{\mathrm{V}} \sin \mathbf{u}_{\mathrm{V}}=\mathbf{n} \sin \mathrm{U}
$$

Cette quantité invariante n sin U est l'ouverture numérique de l'objectif. Plus encore que son grandissement, cette grandeur est sa caractéristique principale. Outre qu'elle régit la quantité de lumière entrant dans l'instrument, c'est elle qui définit sa profondeur de champ et sa propriété la plus essentielle : sa limite de résolution. 


\subsubsection{Limite de résolution de l'objectif}

Doter un objectif d'un grandissement suffisamment fort pour agrandir notablement l'image n'est pas suffisant. Il est encore nécessaire, à l'intérieur des limites de l'objet, d'en agrandir les détails, de les mettre en évidence, de les résoudre. La classique limite de séparation entre deux points n'est pas vraiment significative pour définir la qualité d'un objectif de microscope destiné à l'observation d'objets étendus dont les points peuvent présenter entre eux à la fois un certain degré de cohérence et un déphasage.

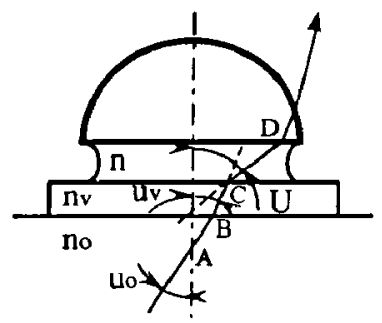

Figure 2 : Définition de l'ouverture numérique

En microscopie, de même qu'en photographie et en astronomie en dehors de l'observation des étoiles, on peut considérer que le champ étendu de l'objet, à l'image d'une photographie tramée, est constitué d'une structure pouvant être décomposée en un ensemble d'objets périodiques de fréquence "spatiale", de contraste et d'orientation différents. La limite de résolution de l'objectif est alors définie comme étant la plus petite période résolue par l'objectif. Cette limite de résolution varie suivant l'état de cohérence de la lumière d'éclairage, c'est-à-dire pratiquement selon les dimensions de la source. Nous allons examiner simplement, nous inspirant des expériences d'Abbe, comment l'ouverture numérique détermine cette limite.

Considérons un objet, dit de phase, dont l'amplitude complexe ne présente dans une direction que des variations de transmission purement sinusoidales de période spatiale $p$. Immergé dans un milieu d'indice $n$, éclairé par une onde plane $\Sigma_{i}$ émise par une source ponctuelle $S$ placée au foyer d'un condenseur $C_{d}$ (fig. 3), il se comporte comme un réseau diffractant la lumière de longueur d'onde $\lambda$ dans deux directions symétriques inclinées sur l'axe d'angles $\pm \alpha$ tel que

$$
\mathrm{n} \sin \alpha=\lambda / \mathrm{p}
$$

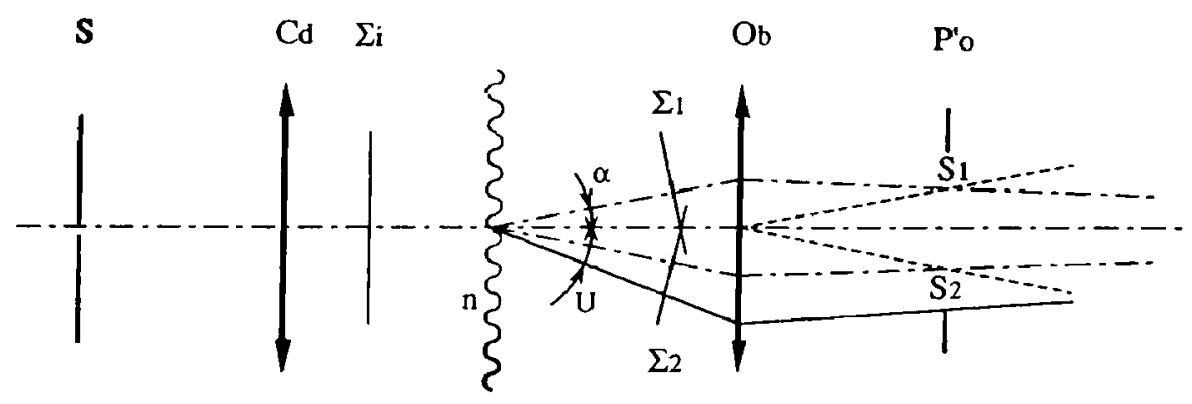

Figure 3 : Définition de la limite de résolution 
Tant que $\alpha$ reste inférieur à $U$, angle du rayon lumineux le plus incliné pénétrant dans l'objectif pouvant en ressortir (angle d'ouverture objet), il en est de même des deux ondes $\Sigma_{l}$ et $\Sigma_{2}$ diffractées par le réseau. L'ensemble condenseur-objectif fournit ainsi dans le plan de la pupille de sortie $P^{\prime}{ }_{o}$ de l'objectif deux images de la source $S_{/}$et $S_{2}$ qui, cohérentes entre elles, reconstruisent par interfërence une image conforme de l'objet. La limite de résolution est dans ce cas d'éclairage cohérent la période $p_{c}$ (fig. 6) telle que

$$
1 / p_{c}=(n \sin U) / \lambda
$$

et, pour toute fréquence spatiale l/p inférieure, l'image obtenue est de contraste unité alors que les fréquences spatiales supérieures ne sont pas transmises. Notons que si l'objet n'est pas de profil sinusoidal, quoique périodique, il est décomposable en un spectre de composantes sinusoïdales fournissant chacune une paire d'ondes diffractées reconstruisant une image conforme tant qu'elles sont transmises par la pupille. $\dot{A}$ la limite, si seules les ondes correspondant à la fréquence spatiale $\lambda p$ la plus basse, c'est-à-dire la fréquence fondamentale correspondant à la période du réseau, sont transmises par la pupille, l'image reconstruite par interférences est purement sinusoïdale.

Inversement si la source est infiniment étendue (éclairage incohérent), elle est néanmoins limitée par l'image dans son plan de la pupille de sortie $P_{o}^{\prime}$, l'ouverture numérique du condenseur se trouvant naturellement limitée à $n \sin U$. Si l'on considère alors un objet dont le facteur de transmission soit sinusoidal en intensité, outre une onde directe remplissant toute la pupille, il fournit deux ondes diffractées auxquelles correspondent deux images $S_{1}$ et $S_{2}$ de la source (fig. 4). Dans les zones communes à deux de ces ondes existent des points homologues pouvant indépendamment fournir des systèmes d'interférences identiques reconstruisant une image de l'objet.

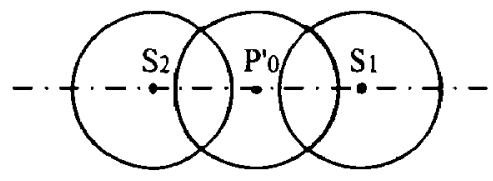

Figure 4 : Images pupillaires de la source en eclairage incohérent

Il y a perte d'énergie et le contraste, proportionnel à l'aire commune aux cercles $S_{I-} P_{o}^{\prime}$ et $S_{2}-P_{o}^{\prime}$, diminuant quand la fréquence spatiale augmente, s'annule pour une fréquence spatiale double de la limite précédente lorsque les images $S_{1}$ et $S_{2}$ sont tangentes extérieurement à $P_{0}^{\prime}$ :

$$
1 / \mathrm{p}_{\mathrm{i}}=(2 \mathrm{n} \sin \mathrm{U}) / \lambda
$$

La période correspondante (fig 6)

$$
\mathrm{p}_{\mathrm{i}}=\lambda /(2 \mathrm{n} \sin \mathrm{U})
$$

est la limite de résolution absolue de l'objectif.

L'amélioration du contraste pour les faibles fréquences spatiales s'obtient en limitant l'ouverture numérique du condenseur à une valeur $\boldsymbol{n} \sin u_{c}$ inférieure à l'ouverture numérique de l'objectif par un diaphragme d'ouverture $D_{o}$ dont limage dans le plan de la pupille de sortie de l'objectif est plus petite que cette pupille (fig 5). 
Par un raisonnement analogue à celui qui a été présenté dans le cas de l'éclairage incohérent, on montre, ce qui avait été constaté depuis fort longtemps par les microscopistes utilisant couramment cette procédure, qu'avec cet éclairage partiellement cohérent, le contraste de l'image (fig. 6) reste égal à l'unité tant que $1 / p<\left(n \sin U-n \sin u_{c}\right) / \lambda$ et s'annule pour une limite de résolution $\mathrm{p}_{\mathrm{s}}$ telle que

$$
1 / p_{s}=\left(n \sin U+n \sin u_{c}\right) / \lambda
$$

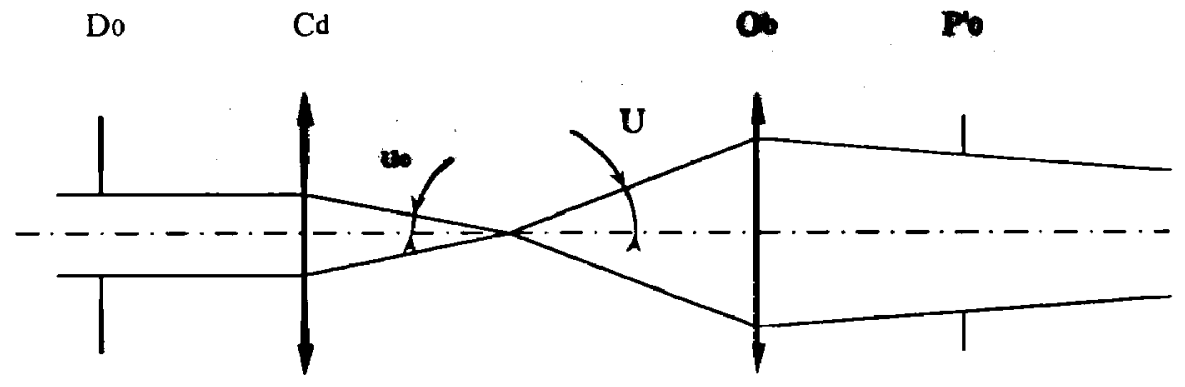

Figure 5 : L'éclairage partiellement cohérent

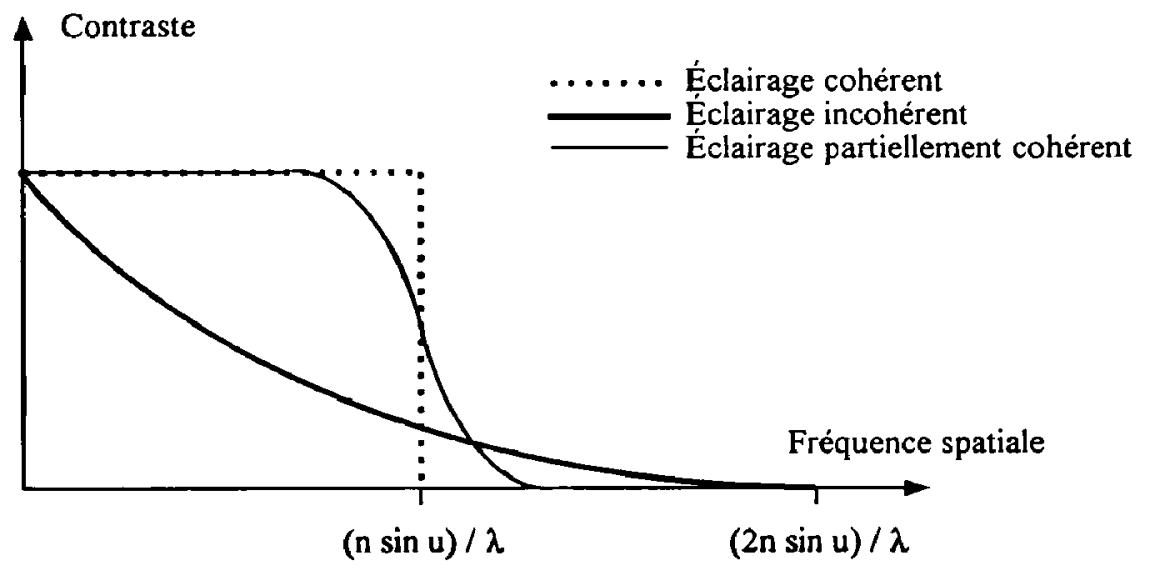

Figure 6 : Variations du contraste d'un objet périodique en fonction de sa fréquence spatiale

Quoiqu'il en soit, la limite de résolution est d'autant meilleure que l'ouverture numérique est plus grande. Il existe quelques rares objectifs à sec $(\mathrm{n}=1)$ d'ouverture 0,95 , soit un angle $U$ exceptionnellement grand de $72^{\circ}$, de limite de résolution absolue égale à $0,3 \mu \mathrm{m}$. L'immersion homogène $(n=1,518$ correspondant à l'indice des lamelles et de la lentille 
frontale) permet des résolutions inférieures à $0,2 \mu \mathrm{m}$ avec des ouvertures de 1,25 correspondant à un angle $U^{\prime}$ de seulement $55^{\circ}$.

\subsection{L'éclairage du microscope}

\subsection{L'éclairage, le besoin et les moyens}

Les objets examinés en microscopie sont rarement lumineux par eux-mêmes et il est nécessaire de les éclairer. Compte tenu de la faible clarté du microscope due au faible diamètre de sa pupille de sortie et à la réduction de son facteur de transmission par le grand nombre de lentilles qu'il comporte, un dispositif d'éclairage est nécessaire pour éclairer uniformément et intensément l'objet.

On dispose à cet effet d'un ensemble source constitué d'une lampe et d'un collecteur, système optique destiné à concentrer le flux émis par la lampe. Les lampes utilisées sont soit à filament de tungstène, généralement en atmosphère halogènée afin d'en accroître la durée de vie avec une luminance constante, soit à décharge à arc court et haute pression en atmosphère de mercure ou de xénon de quelques centaines d'heures de durée de vie, à température de couleur proche de celle du soleil. Le collecteur sert à prélever la plus grande partie du flux fourni par ces sources qui émettent pratiquement dans un angle solide de $4 \pi$ et à le concentrer dans l'ouverture du microscope.

\subsubsection{L'éclairage fond clair}

Ce type d'éclairage où l'objet est directement illuminé est le plus classique en microscopie. La solution la plus simple, longtemps utilisée, consiste à projeter directement la source dans le plan de l'objet à l'aide du collecteur. Cet éclairage critique n'est pas uniforme, l'image du filament étant confondue avec celle de l'objet, et on y remédie en dépointant légèrement la source ou en formant son image dans le plan d'une surface légèrement absorbante faiblement dépolie placée au voisinage du plan objet. Du fait des aberrations du collecteur l'image de chaque point de la source n'est pas ponctuelle et dans ces conditions chaque point de l'objet est éclairé partiellement par plusieurs points de la source ou plus clairement des points voisins de l'objet reçoivent de la lumière provenant d'un même point de la source, ce qui définit un éclairage partiellement cohérent avec les avantages en résolution que cela présente. Cet état est encore plus manifeste avec l'emploi du verre dépoli. Néanmoins, dans cette solution, l'ensemble du flux transmis par le collecteur peut ne pas pénétrer intégralement dans la pupille et ainsi ne pas contribuer entièrement à la formation de l'image. Cette remarque évidente qui s'applique à tout instrument d'optique aussi simple soit-il (l'observation à la loupe d'une diapositive éclairée par une ampoule électrique lointaine est seulement lumineuse lorsque l'image du filament donnée par la loupe se forme dans la pupille de l'œil), justifie l'intérêt de l'éclairage introduit en 1893 par Köhler.

Cet éclairage (fig. 7) consiste à projeter la source $S$ dans le plan de la pupille d'entrée du microscope matérialisée par un diaphragme iris $D_{0}$ placé dans le plan focal objet d'un condenseur $C_{d}$ situé en avant de l'objet. Chaque point de celui-ci recevant alors de la lumière de tous les points de la source est éclairé uniformément de même que l'est le collecteur $C_{l}$. On place donc en son voisinage un second diaphragme iris $D_{c}$ dont le condenseur forme une image dans le plan de l'objet afin d'une part d'en limiter l'étendue et d'autre part surtout de réduire la lumière parasite provenant de la diffusion de lumière par les parties du champ qui 
dépasseraient la capacité de saisie du tube du microscope, ou des réflexions par la paroi de ce tube. En modifiant la dimension du diaphragme $D_{0}$, il est possible de faire varier le degré de cohérence de l'éclairage (cohérent à diaphragme minimum, incohérent à diaphragme pleinement ouvert, partiellement cohérent aux ouvertures intermédiaires).

Les microscopes de type biologique, travaillant en lumière transmise, sont munis d'un tel condenseur. Si sa qualité n'affecte pas l'imagerie, il peut être néanmoins corrigé de certaines aberrations pour qu'il ne fournisse pas d'image trop déformée du diaphragme de champ. Les méthodes de contraste de phase et de contraste interférentiel, que nous évoquons au chapitre suivant, imposant une meilleure correction des aberrations pupillaires, imposent des condenseurs aplanétiques et achromatiques diminuant notamment l'irisation du diaphragme de champ. Dans les microscopes de type métallographique, travaillant en lumière réfléchie, l'objectif sert également de condenseur.

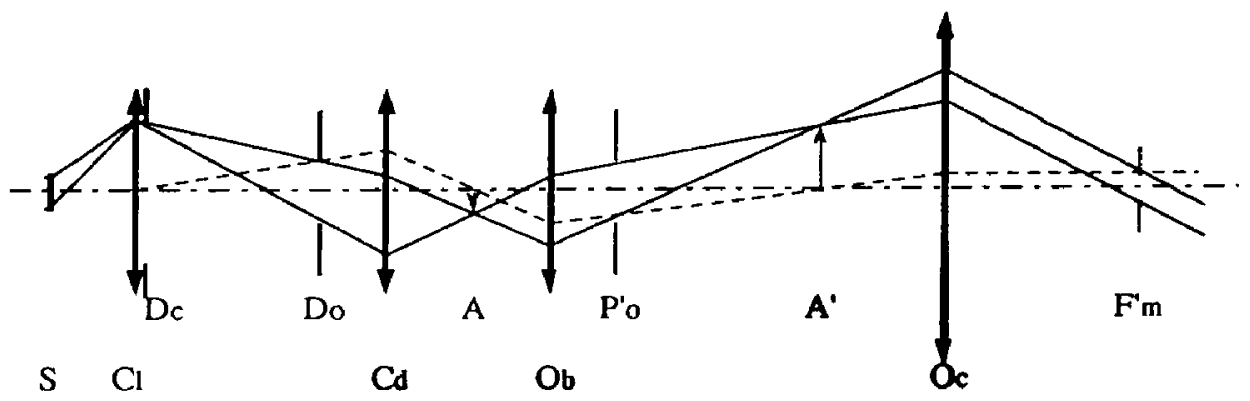

Figure 7 : Schéma de l'éclairage de Kohler

\section{MICROSCOPIE DES OBJETS DE PHASE}

\subsection{L'objet de phase}

Certains objets ne sont pas visibles en microscopie, non parce qu'ils sont de dimensions trop faibles, mais parce qu'étant parfaitement transparents, il n'existe aucune variation d'absorption (ou de réflexion pour les objets réfléchissants) dans leur structure et qu'ainsi leurs différentes zones ne se différencient par aucune variation de contraste. Par contre ils présentent entre leurs points des différences d'épaisseur etou d'indice de réfraction. Lorsqu'une onde lumineuse $\Sigma_{j}$ traverse de tels objets, ou s'y réfléchit, elle parcourt des chemins optiques (produits de l'indice de réfraction par l'épaisseur) variables, suivant un trajet plus ou moins long suivant que le milieu est plus ou moins épais, à une vitesse plus ou moins grande suivant que l'indice est plus ou moins faible.

Ces variations de chemin optique $\Delta$ (fig. 8) se traduisent par des déformations de la surface d'onde transmise $\Sigma\left(\right.$ a) ou réfléchie $\Sigma_{r}$ (b) et sont respectivement égales à $\left[\left(n^{\prime}-n\right) e\right]$ pour un objet transparent d'épaisseur locale $e$, d'indice $n^{\prime}$, immergé dans un milieu d'indice $n$ et $\grave{a} 2 e$ pour un objet réfléchissant. Les surfaces d'onde étant des surfaces équiphases, et la phase variant de $2 \pi$ lorsque la lumière parcourt un chemin optique égal à la longueur d'onde $\lambda$, ces déformations sont équivalentes à des déphasages introduits par l'objet 


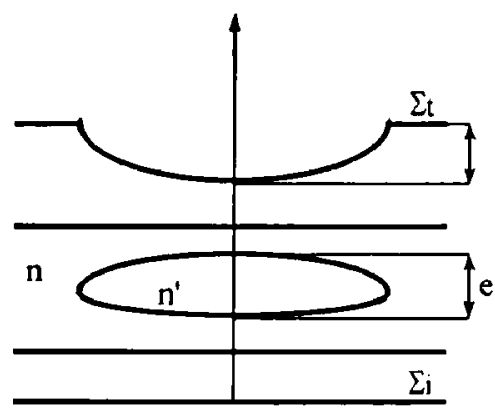

a

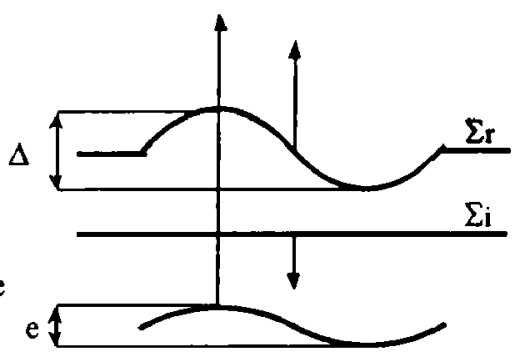

b

Figure 8 : Définition de l'objet de phase

L'examen de tels objets impose la mise en évidence de leurs variations de phase. Des techniques d'observation comme le contraste de phase et l'interférométrie permettent d'atteindre ce but.

\subsection{Le contraste de phase}

\subsection{Principe du contraste de phase}

Cette méthode a été imaginée en 1934 par le hollandais Zernicke et lui valut le prix Nobel de Physique en 1953. En les transformant en variations d'intensité, les variations de phase sont rendues visibles par cette méthode. Supposons (fig. 9) une source ponctuelle $S$ au foyer d'un condenseur achromatique et aplanétique. Le faisceau issu de la source fournit une onde plane qui traverse l'objet. Si l'on admet l'objet vide, après traversée de l'objectif, cette onde, devenue sphérique, converge en $S^{\prime \prime}$ dans le plan de la pupille de sortie et éclaire uniformément le plan de l'image intermédiaire. C'est le faisceau de lumière directe. S'il existe dans l'objet un détail déphasant $B$ non dénué de dimension, il diffracte la lumière dans un angle d'autant plus grand qu'il est de plus faible dimension. Le faisceau de lumière diffractée s'étale dans le plan de la pupille et vient converger en $B^{\prime}$ image de $B$

Les deux ondes directe et diffractée, issues d'un même point source sont cohérentes entre elles et peuvent donc interférer. L'image observée en $\mathrm{B}^{\prime}$ résulte donc des interférences entre ces deux ondes et, dans un diagramme de Fresnel, peut être représentée (fig. 10a) par un vecteur $\mathbf{O B}^{\prime}$ ' de même norme (puisqu'il n'y a pas variation d'absorption et si l'on suppose que l'objectif recueille toute la lumière diffractée) que celle du vecteur $\mathbf{O A}^{\prime}$ représentant l'onde directe, déphasé de l'angle $\phi . \mathbf{O B}$ ' résultant donc des interférences entre onde directe $\mathbf{O A}$ ' et onde diffractée $\mathbf{D}$, on vérifie

$$
\mathbf{O B}^{\prime}=\mathbf{O A}^{\prime}+\mathbf{D}=\mathbf{O A}^{\prime}+\mathbf{A}^{\prime} \mathbf{B}^{\prime}
$$


et le vecteur $\mathbf{A}^{\prime} \mathbf{B}^{\prime}=\mathbf{D}$ représente donc l'onde diffractée et si nous considérons que le déphasage est très faible, on peut admettre que les vecteurs $\mathbf{O A}^{\prime}$ et $\mathbf{A}^{\prime} \mathbf{B}^{\prime}$ représentant respectivement les ondes directe et diffractée sont en quadrature de phase.

Supposons alors que l'on place en $S^{\prime}$ image de $S$ (fig. 9) une lame recouvrant l'image de la source, d'indice et d'épaisseur tels qu'elle avance ou retarde de $\pi / 2$ (lame quart d'onde) l'onde directe. Elle n'affecte pratiquement que cette onde et le vecteur $\mathbf{O A}^{\prime}$ devient (fig. $10 \mathrm{~b}$ ), suivant qu'elle avance ou retarde, $\mathrm{O}_{1} \mathrm{~A}^{\prime}$ ou $\mathrm{O}_{2} \mathrm{~A}^{\prime}$, pratiquement en phase ou en opposition de phase avec le vecteur $\mathbf{A}^{\prime} \mathbf{B}^{\prime}$. Le vecteur résultant des interférences entre les deux ondes, en supposant que la norme de $\mathbf{O A}^{\prime}$ (et de $\mathbf{O B}^{\prime}$ ') soit égale à l'unité, est alors de norme égale à $(1 \pm \phi)$ et l'intensité en $B^{\prime}$ est égale à $(1 \pm \phi)^{2} \approx 1 \pm 2 \phi$ alors que celle du fond est égale à l'unité. Son contraste par rapport au fond, défini comme le rapport de la différence des intensités maximale et minimale à leur somme, est donc égal à $\pm \phi$.

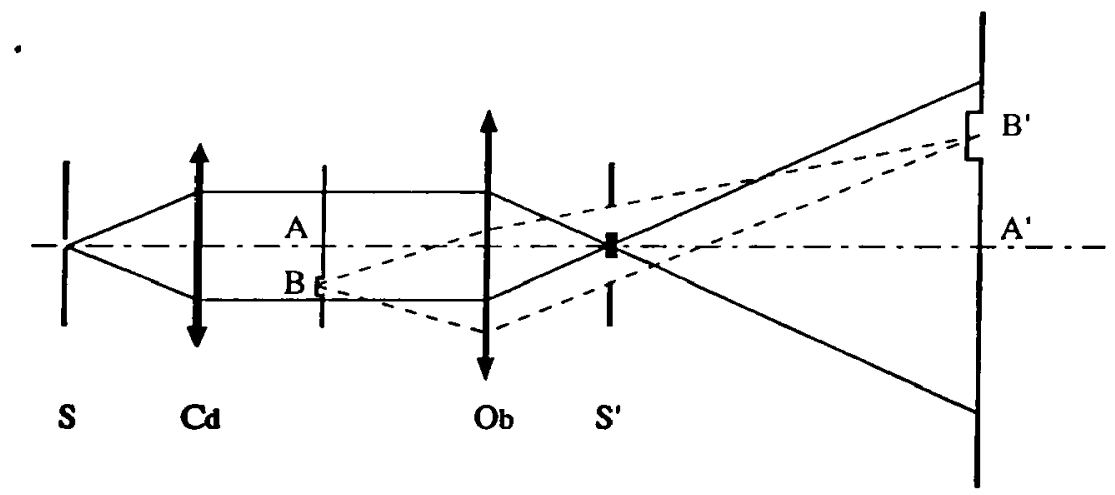

Figure 9 : Schéma du contraste de phase
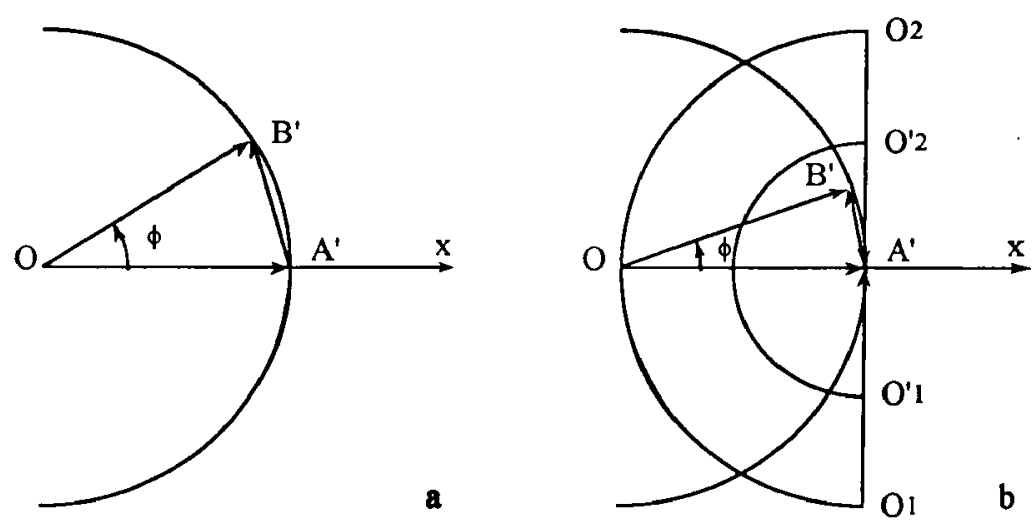

Figure 10 : Diagrammes de Fresnel expliquant le principe du contraste de phase 
Si la lame de phase avance $(+)$ l'onde directe d'un quart d'onde (épaisseur optique $32 / 4$ ), à une avance de phase $(\phi>0)$ correspond une augmentation d'éclairement, à un retard de phase $(\phi<0)$ une baisse d'éclairement, c'est le contraste de phase positif. Inversement, si la lame de phase retarde (-) l'onde directe d'un quart d'onde (épaisseur optique $2 / 4$ ), à une avance de phase correspond une baisse d'éclairement et vice versa, c'est le contraste de phase négatif. Pour améliorer la sensibilité de la méthode, le contraste restant faible, la lame de phase est également absorbante. De facteur de transmission en amplitude $t<1$ (donc de facteur de transmission $t^{2}$ en intensité), son but n'est pas seulement de faire tourner le vecteur $\mathbf{O A}^{\prime}$, mais encore de réduire sa norme à la valeur $t$ (ce qui amène $O$ en $O_{1}^{\prime}$ ou $O_{2}^{\prime}$ ) sans affecter celle de A'B'. Dans ces conditions l'intensité en $B^{\prime}$ devient

$$
\mathrm{I}_{\mathrm{B}^{\prime}}=(\mathrm{t} \pm \phi)^{2} \approx \mathrm{t}^{2} \pm 2 \mathrm{t} \phi
$$

et quoique le niveau moyen d'éclairement soit diminué, le contraste égal à $\pm \phi / t$ est amélioré.

\subsubsection{Le contraste de phase variable}

Avancer ou retarder l'onde directe d'un quart d'onde n'est qu'une solution approchée favorable aux faibles déphasages. Pour des valeurs notables, $\mathbf{O A}^{\prime}$ et $\mathbf{A}^{\prime} \mathbf{B}^{\prime}$ ne peuvent être considérés en quadrature et pour que l'éclairement de l'image de l'objet de phase soit maximal ou minimal par rapport à celui du fond, l'onde directe $\mathbf{O A}^{\prime}$ doit être amenée en phase ou en opposition de phase avec l'onde diffractée $\mathbf{A}^{\prime} \mathbf{B}$ ' et venir dans le diagramme de Fresnel (fig. 11 a) occuper la position $\mathbf{O A}_{1}^{\prime}$ ouOA' ${ }_{2}$, c'est-à-dire subir les déphasages $\psi_{1}$ ou $\psi_{2}$ égaux à $\pm \pi / 2+\phi / 2$. Le contraste est alors optimal. Il est égal à l'unité si l'éclairement est nul en $B^{\prime}$, ce qui nécessite (fig. $11 \mathrm{~b}$ ) d'absorber l'onde directe avec une lame de facteur de transmission en amplitude $t=2 \sin / \phi / 2 /$.
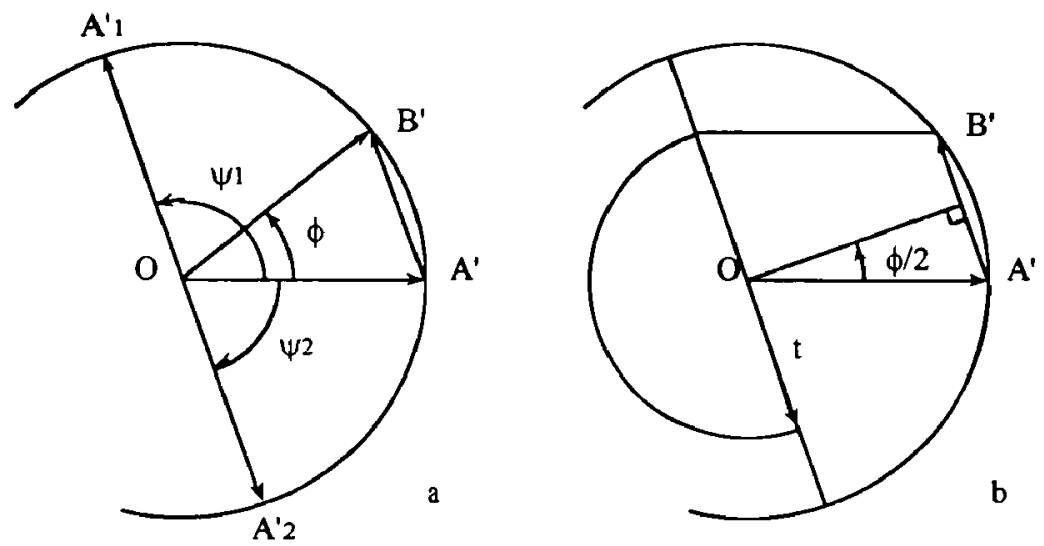

Figure 11 : Diagrammes de Fresnel expliquant le principe du contraste de phase variable

Il existe des contrastes de phase variables répondant à la question. Cette terminologie est à double sens: ils s'adaptent à la phase variable de l'objet et, pour une phase donnée, il est 
possible de faire varier le contraste de l'image. Ces dispositifs, au catalogue de rares constructeurs, sont fondés sur l'emploi de composants travaillant en lumière polarisée, dont les orientations relatives permettent d'adapter déphasage et absorption, et ainsi de mesurer la phase puisque ces paramètres en sont fonction (voir encadré). Ce sont les seuls dispositifs permettant cette procédure, le contraste de phase n'étant en général qu'un procédé de visualisation et non de mesure.

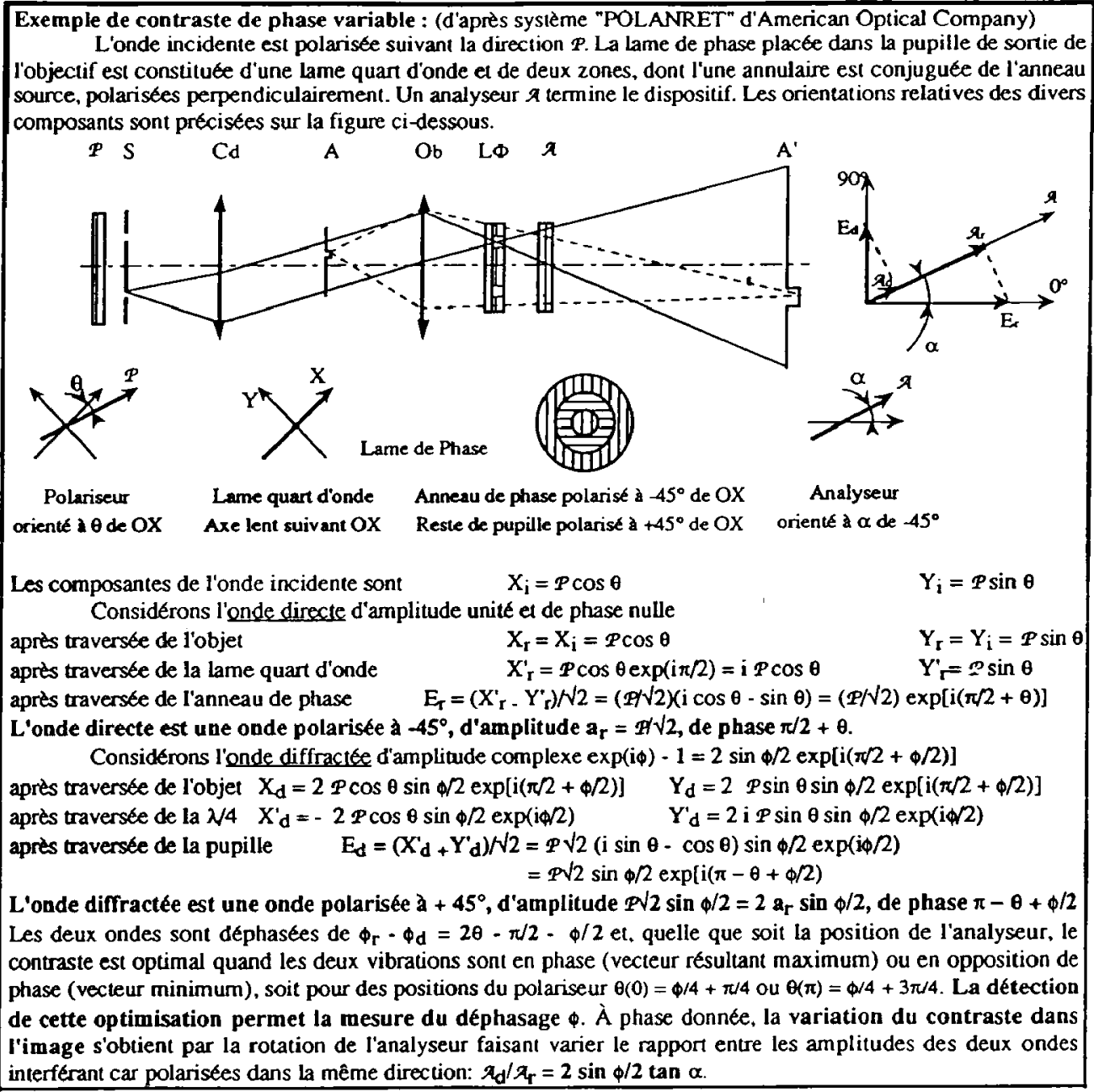

\subsubsection{Réalisation du contraste de phase}

On n'utilise pas une source lumineuse et une lame de phase ponctuelles, ou centrales de faibles dimensions, pour réaliser pratiquement les dispositifs à contraste de phase, la diffraction de 
l'onde directe par la lame de phase diminuant la qualité de l'image en produisant des franges sur ses bords. Ȧ surface égale, ce phénomène de halo est moins important avec une lame de phase et une source annulaires, et c'est sous cette forme que les dispositifs à contraste de phase sont réalisés. Le plan de la pupille de sortie de l'objectif contenant la lame étant souvent interne à la combinaison, cela nécessite la conception d'objectifs spéciaux à lame incorporée, déposée sur l'un des dioptres du système (une couche diélectrique déphasante, une couche métallique absorbante). À chaque objectif est associé un anneau source centrable, l'ensemble de ceux-ci étant monté dans une tourelle tournante dans le plan focal objet du condenseur. Centrage et conjugaison des anneaux source et de phase se contrôlent en observant la pupille à l'aide d'une lunette constituée de l'oculaire et d'une lentille additionnelle. En métallographie, cette solution n'est pas possible, l'objectif servant également de condenseur. On adopte un schéma, tendant d'ailleurs à s'adapter aux équipements biologiques, consistant à doter le statif d'un véhicule pupillaire conjuguant la pupille des objectifs d'un revolver porteur des lames de phase, un anneau source commun étant placé dans l'illuminateur.

\subsection{L'interférométrie en microscopie}

\subsection{Généralités sur l'interférométrie}

Les déformations apportées à la surface d'onde par l'objet de phase peuvent être mises en évidence, et éventuellement mesurées, par interférométrie. Suivant son mode de fonctionnement, cette dernière peut être classée en trois catégories recevant toutes, application en microscopie.

En interférométrie normale (fig. 12 a), l'onde incidente $\Sigma_{i}$ est totalement dédoublée et les deux ondes obtenues cheminent suivant deux trajets entièrement distincts, l'objet étant placé sur l'un d'entre eux. Après recombinaison, l'onde $\Sigma_{0}$ empreinte des déformations introduites par l'objet et l'onde de référence $\Sigma_{\boldsymbol{r}}$ interfèrent et la différence de marche variable due à l'objet est mise en évidence et mesurable. La séparation totale des deux ondes impose des statifs spéciaux que l'on ne rencontre pratiquement plus qu'en métallographie.

En interférométrie à double foyer (fig. 12 b), l'onde incidente est dédoublée longitudinalement. Il en est de même de l'image de l'objet donnée par le microscope, mais seule une de ces images est au point. La phase de l'onde correspondante $\Sigma_{0}$ est modifiée par les variations locales d'épaisseur optique, alors que l'effet sur l'autre onde $\Sigma_{r}$, servant d'onde de référence, est celui de l'épaisseur optique moyenne de l'objet e $i$ de l'espace environnant. L'interférogramme obtenu est comparable à un interférogramme normal. Rarement produits suivant une conception fragile, les interféromètres de ce type ont disparu des catalogues mais ils conservent l'intérêt d'être à l'origine du modèle d'interféromètre différentiel le plus répandu.

En interférométrie différentielle (fig. $12 \mathrm{c}$ ), après dédoublement latéral de l'onde incidente en deux ondes subissant l'influence de l'objet, leur recombinaison fournit un interférogramme mettant en évidence la différence de chemin optique entre deux points de l'objet séparés par une distance égale au dédoublement. Ce type d'interféromètre, presque essentiellement construit sous une forme unique, est le plus utilisé pour la visualisation des objets de phase. 


\subsubsection{L'interféromètrie normale}

En microscopie par transmission, on s'est inspiré de l'interféromètre de type Mach-Zehnder, deux microscopes complets identiques étant placés sur les deux bras de l'interféromètre (fig. 13). Cet instrument n'a pas vraiment connu de réel développement, probablement par la difficulté de compensation de ses deux trajets. L'un des microscopes examine l'objet $A$, l'autre, à titre de référence, un objet $R$ vide, ou tout au moins d'épaisseur optique du même ordre en moyenne que celle de l'objet. Différents compensateurs, d'épaisseur optique variable par translation ou rotation, permettent l'égalisation des chemins optiques pour travailler en teinte plate, le basculement relatif des deux ondes pour établir un système de franges d'égale épaisseur, la mesure des variations de chemin optique par modification de teinte ou déplacement de franges.
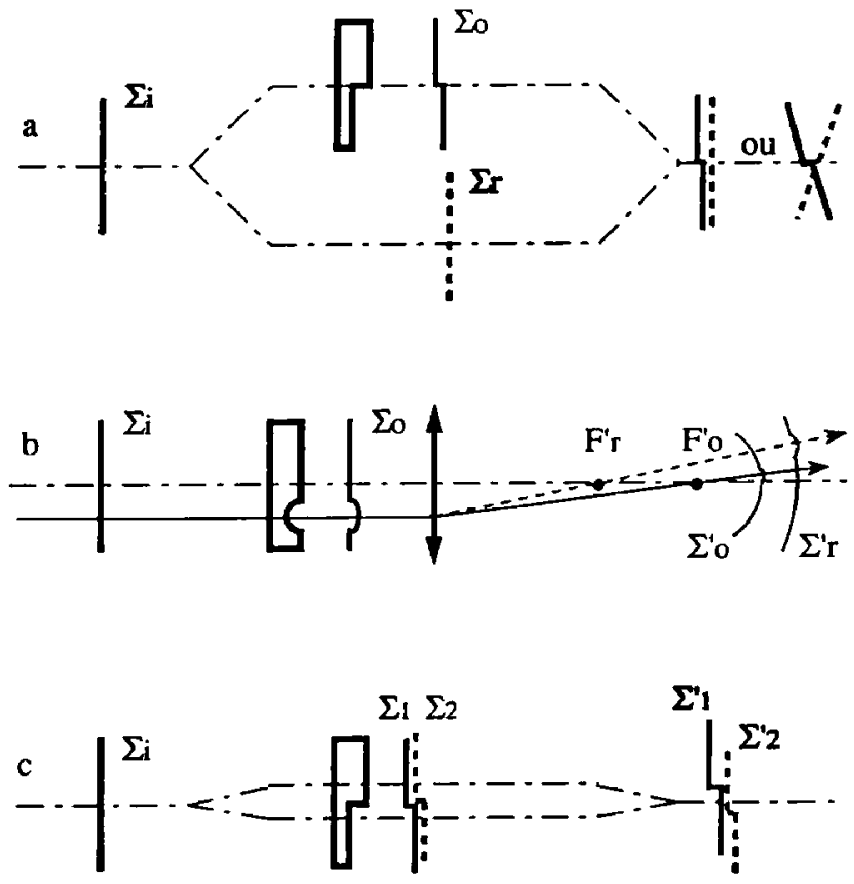

Figure 12 : Les différents types d'interfèrométrie

En microscopie par réflexion, on utilise un schéma de type Michelson introduit par

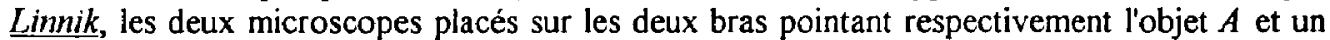
miroir plan de référence $R$ (fig. 14). Compensation et réglages sont obtenus par un ensemble de compensateurs et une platine basculable. Ce type d'interféromètre existe également sous forme d'accessoire adaptable à l'objectif du microscope selon des schémas (fig. 15) dûs à Watson (a) ou à Mirau (b). Leur résolution est limitée par leur distance frontale notable.

L'interférométrie normale peut également être à ondes multiples. Cette solution, due à Tolansky, consiste à placer l'objet à l'intérieur d'un interféromètre de Fabry-Perot. Elle est 
applicable tant en transmission qu'en réflexion, l'un des miroirs de l'interféromètre étant alors l'objet lui-même.

\subsubsection{L'interférométrie à dédoublement longitudinal}

Ce type d'interféromètre est mis en œuvre avec l'interféromètre à double réfraction (ou $\dot{a}$ polarisation) le plus anciennement connu, décrit par Jamin en 1868 et appliqué à la microscopie par Lebedev en 1930.

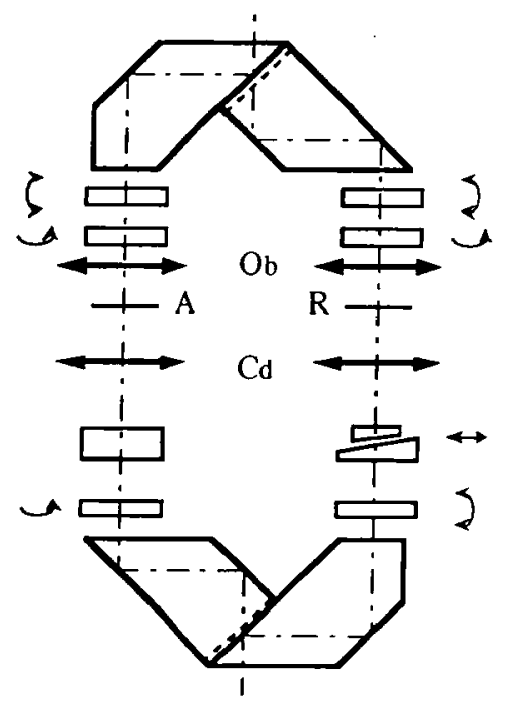

Figure 13 : Micro-interférométrie normale par transmission

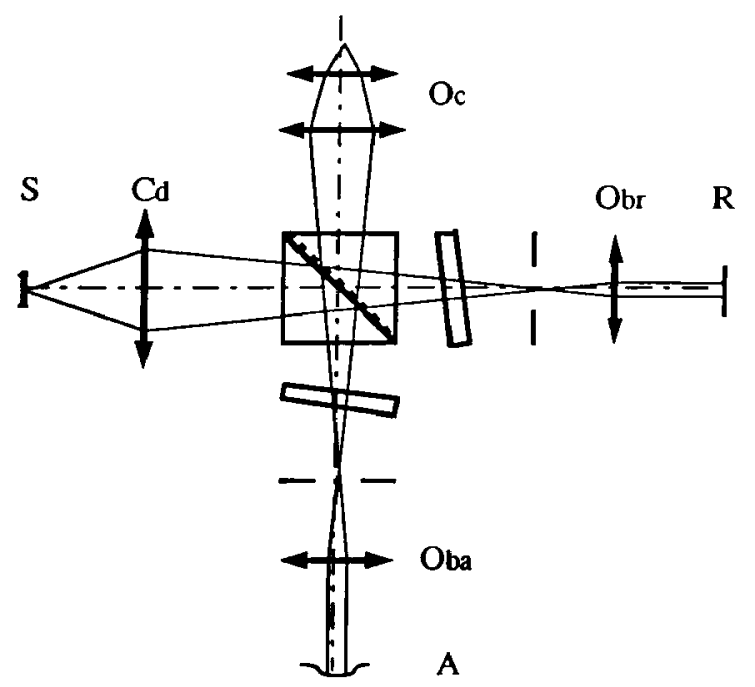

Figure 14 : Micro-interférométrie normale par réflexion selon Linnik 


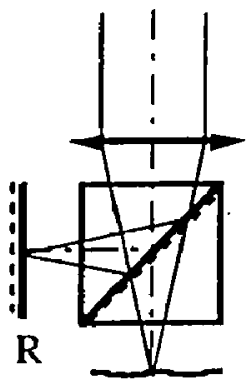

a
$\mathrm{Ob}$

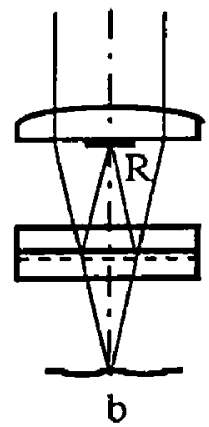

Figure 15 : Micro-interférométrie normale par réflexion selon Watson (a) et Mirau (b)

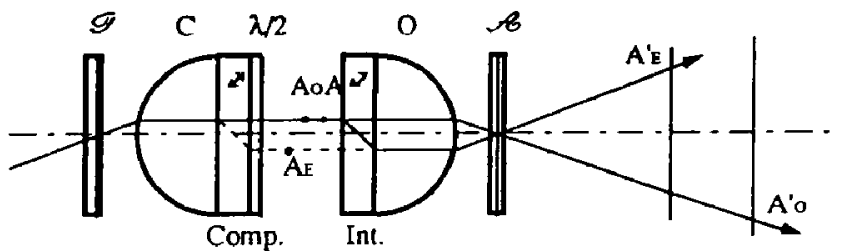

Figure 16: Micro-interférométrie à dédoublement longitudinal selon Jamin - Lebedev

Constitué d'une lame à faces parallèles d'un cristal biréfringent (spath d'Islande) d'axe optique incliné à $45^{\circ}$ sur les faces (fig. 16) placée devant l'objectif $O_{b}$, il donne de l'objet $A$ une image ordinaire $A_{O}$ au point pour l'objectif et une inage extrac slinaire $A_{E}$ dépointée et décalée latéralement, fortement (et fondamentalement) astigmate. L'onde ordinaire correspondante porte l'empreinte des déphasages introduits par l'objet. Il en est de même de l'onde exiraordinaire qui est d'autre part très déformée par l'astigmatisme et suffisamment dépointée pour que chaque point de l'objet "voie" l'ensemble de cette onde. Grâce à cette référence commune à tous les points de l'objet, l'interférogramme obtenu est normal. Les deux ondes, ordinaire et extraordinaire, sont polarisées perpendiculairement par l'action d'un polariseur $\mathscr{S}$ orienté à $45^{\circ}$ du plan de section principale du cristal. Les interfërences sont possibles en repolarisant les deux ondes dans le même plan à l'aide d'un analyseur croisé avec le polariseur. Au déphasage introduit par l'objet s'ajoute celui produit par l'interféromètre entre les deux ondes. Très grand par l'épaisseur du cristal, choisie pour augmenter le dédoublement, et variable avec l'inclinaison des ondes provenant d'une source étendue, l'interférogramme n'est visible que si les déphasages propres à l'interféromètre sont compensés par l'ensemble, placé en avant de l'objet, d'une lame cristalline identique à l'interféromètre, produisant donc le même déphasage entre les deux ondes, accompagnée d'une lame demi-onde d'axe lent orienté à $45^{\circ}$ du plan de section principale commun aux deux cristaux dont l'effet est d'échanger les directions de polarisation des deux ondes. 


\subsubsection{L'imerféroniétrie différemielle}

Le dédoublement latéral entre les deux ondes s'obtient à l'aide d'un interféromètre à deux ondes polarisées ou non, la première solution étant de loin la plus répandue.

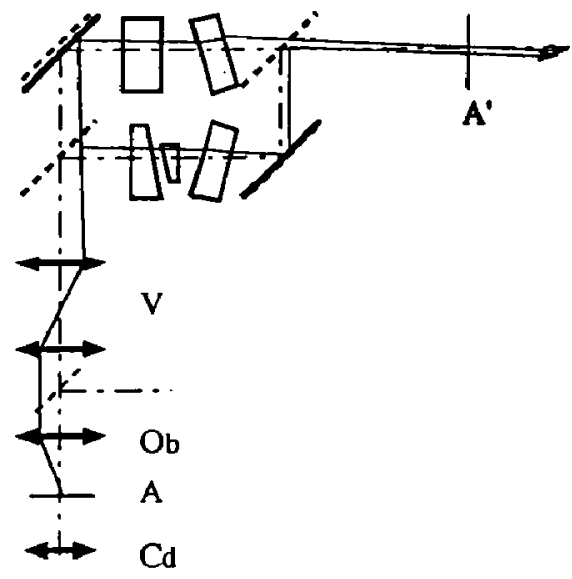

Figure 17 : Micro-interférométrie différentielle basée sur un schéma d'interféromètre de Mach - Zehnder

En ondes non polarisées, on utilise un interféromètre de Mach-Zehnder placé dans l'espace intermédiaire (fig. 17). Des lames inclinables symétriquement sur les deux bras produisent le décalage latéral variable entre les deux ondes, la compensation étant assurée par une lame d'épaisseur optique variable. La présence de l'interféromètre dans cet espace impose l'usage d'un véhicule $V$ assurant le transport des plans pupillaire et objet. Ce dispositif utilisable en lumière transmise ou réfléchie n'est malheureusement plus développé malgré l'intérêt de son dédoublement variable.

Le système le plus généralement utilisé fonctionne en ondes polarisées et est adaptable à un statif de microscope ordinaire. Il est évident que l'interféromètre de Jamin-Lebedev décrit au paragraphe précédent répond à la question puisque produisant un dédoublement latéral des deux ondes. En réduisant suffisamment l'épaisseur de ses cristaux, ce dédoublement latéral peut être exploitable sans que le défaut de mise au point de l'image extraordinaire soit intolérable. Néanmoins cette solution présente certains inconvénients. Les cristaux placés dans l'espace objet limitent par leur épaisseur l'ouverture numérique des objectifs et imposent le calcul d'objectifs spéciaux dont la correction tienne compte de cette épaisseur. Pour la réduire, l'emploi d'un cristal de forte biréfringence (différence entre les indices de réfraction extraordinaire et ordinaire) tel le spath est un avantage, mais ce cristal est particulièrement fragile car clivant aisément sous un choc et son emploi dans un espace libre n'est pas recommandé à cause de son caractère hydrophile. Enfin l'astigmatisme de l'image reste un défaut gênant. Pour remédier en partie à ces défauts, Françon a proposé un oculaire interférentiel où l'interféromètre est un polariscope de Savart constitué de deux lames de Jamin croisées séparées par une lame demi-onde, qui a pour avantages de travailler dans un espace mieux protégé et de rendre les deux images au moins également astigmates.

La solution la plus répandue (fig. 18) dérive de l'emploi d'un biprisme de Wollaston $W_{i}$ placé entre polariseurs croisés orientés à $\pm 45^{\circ}$ de ses axes (afin que les amplitudes des deux 
ondes soient égales et que le contraste de leur figure d'interférences soit égal à l'unité). Situé dans le plan de la pupille de sortie de l'objectif (son plan focal image), il produit deux ondes polarisées perpendiculairement décalées angulairement dans la direction perpendiculaire à l'arête du prisme de

$$
\varepsilon=2 \Delta \mathrm{n} \tan \alpha_{\mathrm{i}}
$$

$\Delta n=n_{e}-n_{O}$ étant la biréfringence du cristal (souvent du quartz) et $\alpha_{i}$ l'angle du prisme.

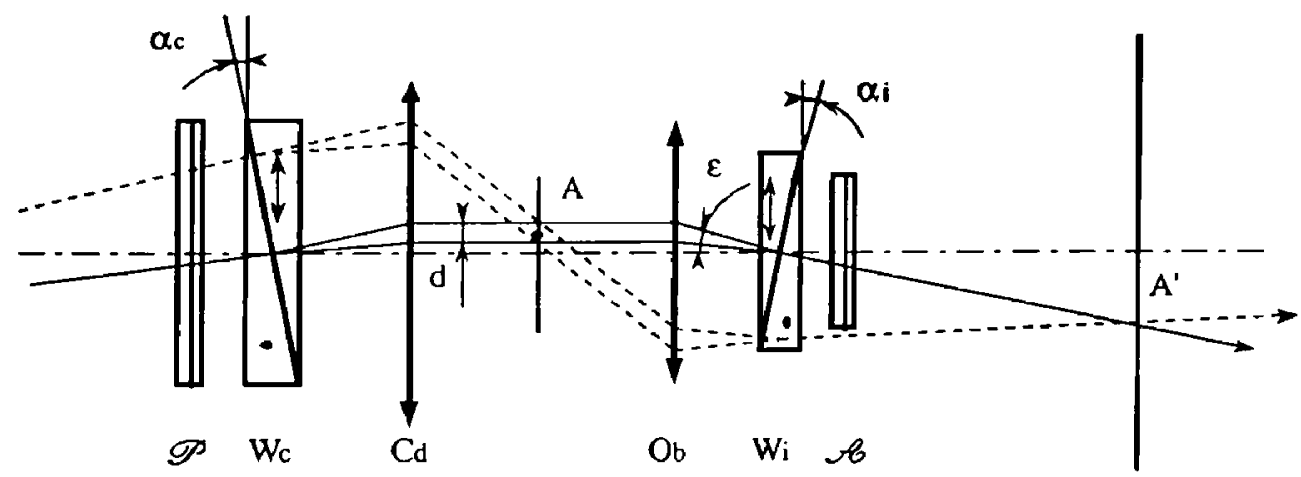

Figure 18 : Micro-interférométrie interférentielle selon Smith - Nomarski

Ce décalage angulaire correspond dans l'espace objet à un décalage latéral des ondes $d=f^{\prime} o b \varepsilon$ La compensation du déphasage variable introduit par l'obliquité des faisceaux (emploi d'une source large), l'interféromètre étant traversé à des hauteurs différentes, est obtenue en plaçant un biprisme compensateur $W_{c}$ dans le plan de la pupille d'entrée du condenseur, d'angle $\alpha_{\mathcal{C}}$ tel que

$$
f_{c d} \tan \alpha_{c}=f_{o b} \tan \alpha_{i}
$$

Cette conjugaison demande un condenseur de haute qualité et est difficile à réaliser pour de larges dédoublements pour lesquels un éclairage en fente est préférable, sauf en microscopie par réflexion où l'interfèromètre est autocompensé, son image par autocollimation sur l'objet (fig. 19) étant au grandissement -1

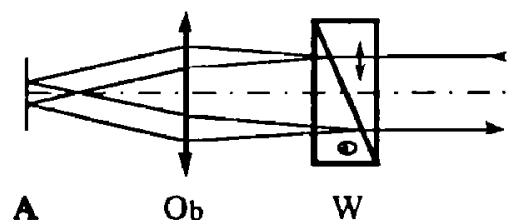

Figure 19 : Micro-interférométrie interférentielle par transmission

Ce système simple proposé par Smith, ne peut pas être utilisé avec les objectifs forts dont le plan pupillaire est interne à lá combinaison. Afin que le plan de dédoublement angulaire des ondes (ou plan de localisation des franges propres à l'interféromètre) soit confondu avec le 
plan de la pupille, Nomarski a proposé un biprisme dérivé du Wollaston (fig. 20), à prismes d'inégales épaisseurs dont l'un est à axe incliné, permettant de sortir ce plan de localisation. Cette solution est aujourd'hui adoptée par la grande majorité des constructeurs.

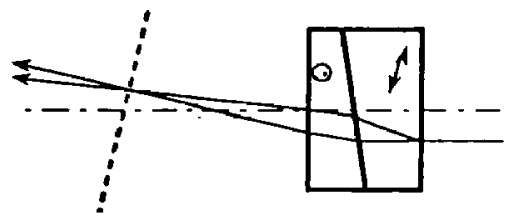

Figure 20 : Biprisme de Nomarski dérivé de celui de Wollaston

Dans cette position (plan de dédoublement dans le plan de la pupille), l'interférométrie est en teinte plate (les ondes ne sont pas basculées dans l'espace objet) qu'il est possible de modifier, en agissant donc sur la compensation, par translation de l'interféromètre dans la direction du dédoublement. Par contre, par translation le long de l'axe optique de la quantité $L$, il produit un dédoublement latéral $a$ de la pupille, donc de la source, égal à $L \varepsilon$, ce qui entraîne un basculement $\beta$ des ondes (fig. 21). Un système de franges d'égale épaisseur s'établit alors dans le plan de l'objet, dans la direction du dédoublement, d'interfrange.

$$
i=\lambda f_{O b} / L \varepsilon
$$

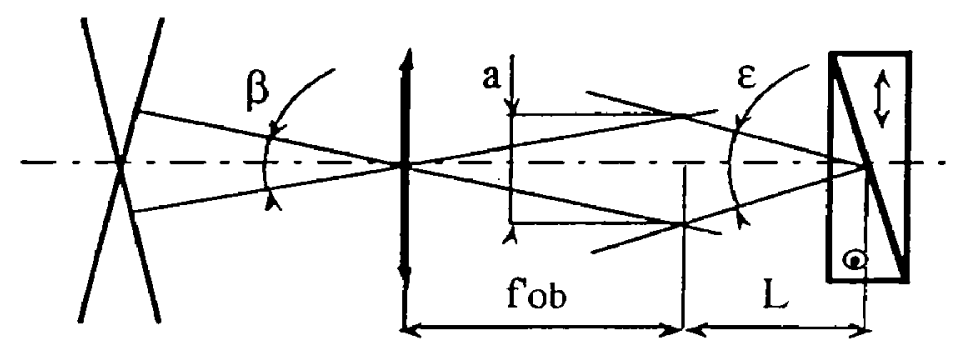

Figurc 21 : Obtention de franges d'égale épaisseur par translation longitudinale de l'interféromètre

Cette interférométrie se prête à deux applications distinctes suivant la valeur du dédoublement latéral produit.

2.3.4.1 Interférométrie à large dédoublement : Considérons des objets isolés, de dimensions latérales inférieures au dédoublement, dont les variations de chemin optique provoquent sur l'onde transmise des déformations $\delta$. L'interféromètre en fournit deux images (fig. 22). En dehors de celles-ci (fond), la différence de marche $\Delta$ entre les deux ondes $\Sigma_{1}$ et $\Sigma_{2}$ est celle qui est éventuellement introduite par l'interféromètre s'il est ou non volontairement mal compensé. 


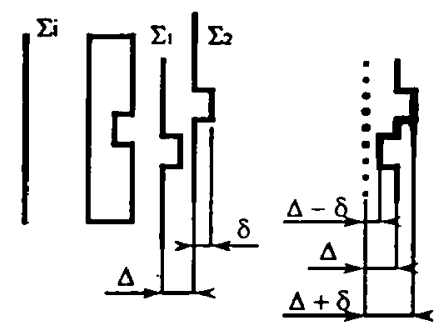

Figure 22 : Interférométrie à large dédoublement latéral

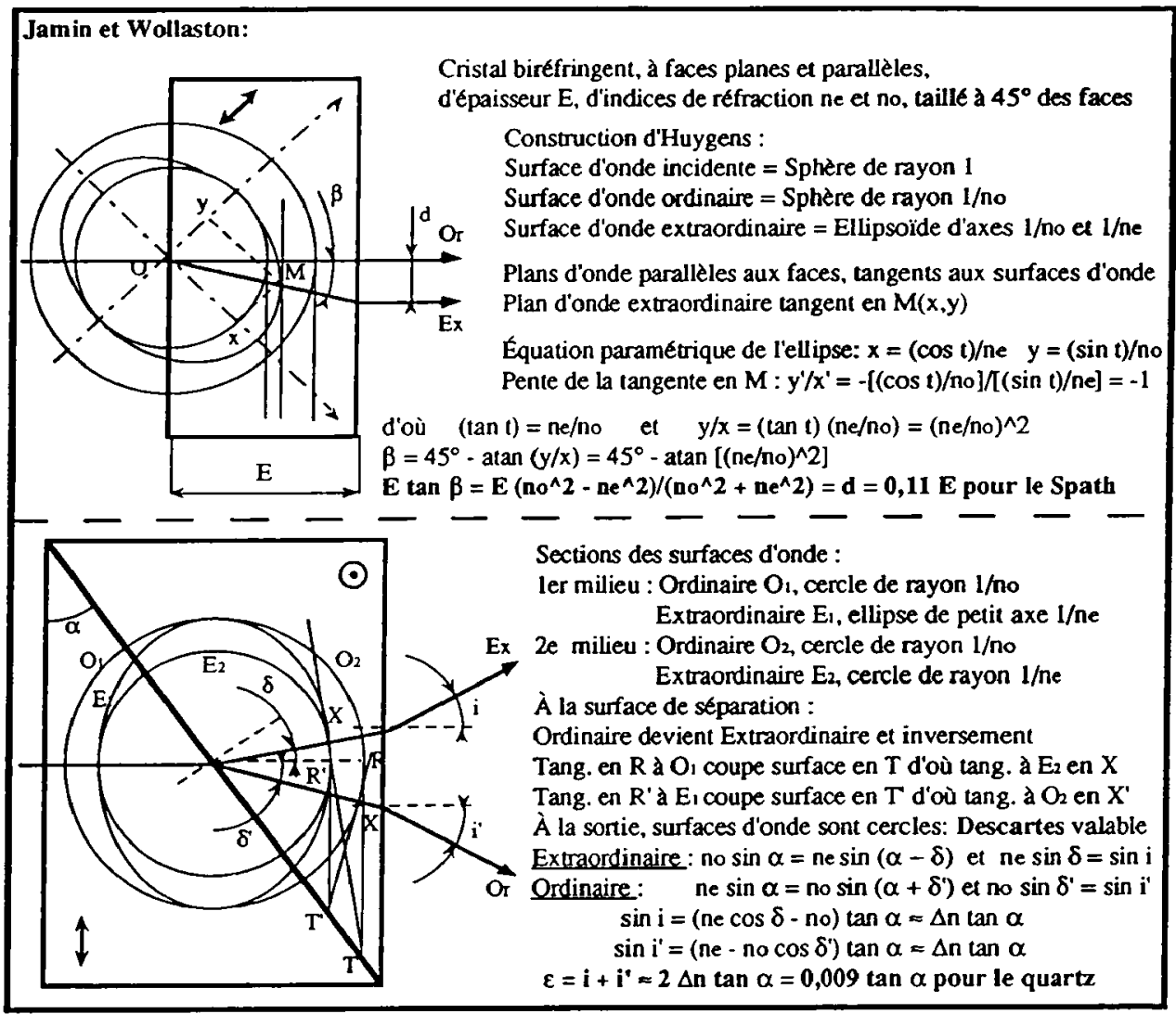

Entre polariseurs croisés, interférences dites soustractives, l'interféromètre met en évidence la différence entre les deux ondes. Pour les deux images, les différences de marche sont donc $\Delta+\delta$ et $\Delta-\delta$. Leurs intensités sont respectivement $\sin ^{2}[(\pi / \lambda)(\Delta+\delta)]$ et $\sin ^{2}[(\pi / \lambda)(\Delta-\delta)]$ alors qu'elle est $\sin ^{2}(\pi \Delta / \lambda)$ pour le fond. Si, à l'aide d'un compensateur auxiliaire, on amène l'intensité du fond à être nulle $(\Delta=0)$, celles des deux images sont égales. $\mathrm{Si}$, à partir de cette situation, on annule successivement ces deux intensités par déplacement du compensateur, la différence entre ses deux positions correspondantes permet la mesure de $2 \delta$. 
L'interférométrie est dans ce cas normale, à ceci près que, grâce aux deux images, sa sensibilité est doublée.

Pour effectuer ces mesures en teinte plate, les deux ondes étant polarisées perpendiculairement, on peut utiliser un compensateur biréfringent de type Babinet, un analyseur à lame quart d'onde (attribué à de Sénarntont), ou même la translation dans son plan de l'interféromètre, mais avec une sensibilité d'autant moins bonne que l'angle du Wollaston est plus grand. En interférométrie à franges d'égale épaisseur, il suffit de pointer à l'aide d'un oculaire micrométrique le double décalage de la frange achromatique obtenue en lumière blanche (fig. 23 a) après étalonnage préalable de sa graduation par rapport à l'interfrange obtenu en lumière monochromatique de longueur d'onde $\lambda$ (fig. 23 b).
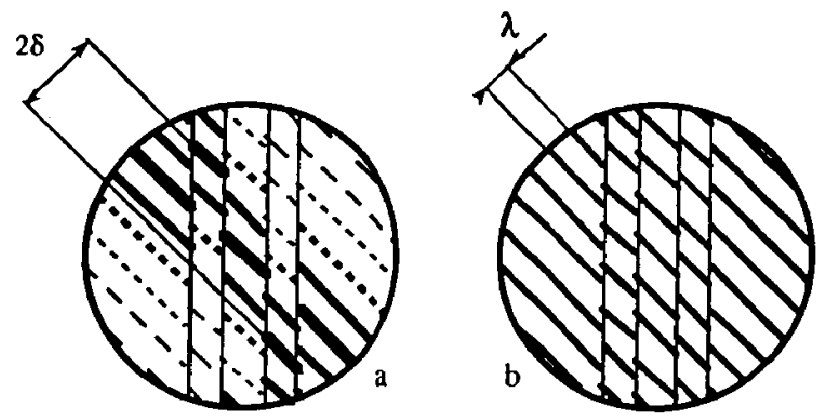

Figure 23 : Mesure d'une différence de marche en lumière blanche (a) après étalonnage en lumière monochromatique (b)

Cette méthode n'est plus utilisable si l'objet est étendu, l'interprétation de l'interférogramme devenant difficile.

2.3.4.2 Contraste interférentiel : Pour les objets étendus de structure complexe, cette interférométrie est néanmoins une méthode de visualisation très appréciée, sous réserve que le dédoublement soit infiniment petit et en toute rigueur inférieur à la limite de résolution de l'objectif afin que le dédoublement de l'image ne soit pas visible.

Pour un dédoublement $d$ dans la direction $x$ (fig. 24), en tout point introduisant une variation de chemin optique $\delta(x)$, la différence de marche $\Delta(x)$ entre les deux ondes est

$$
\Delta(x)=\delta(x+d / 2)-\delta(x-d / 2) \approx d \delta^{\prime}(x)
$$

où $\delta(x)$ représente le gradient (la dérivée) de $\delta(x)$. La différence de marche mise en évidence par interférences est donc une fonction linéaire de la pente de la différence de marche introduite par l'objet. On visualise donc les variations du gradient de la phase et, sauf cas particulier d'une pente constante sur une zone étendue, la mesure n'est plus possible. Cette méthode, très sensible, est concurrente et parfois complémentaire du contraste de phase. Il y a lieu de noter que l'effet directif propre au dédoublement peut nécessiter des examens de l'objet suivant plusieurs orientations par rapport à ce dédoublement pour éviter toute perte d'information. 
En pratique, selon le schéma instrumental de la figure 18, le biprisme interféromètre produit un dédoublement $d=\mathrm{f}_{\circ b} \varepsilon=\lambda / 4 \mathrm{n} \sin \mathrm{U}$ de l'ordre de la moitié de la limite de résolution absolue de l'objectif. Il est donc de dédoublement angulaire $\varepsilon=\lambda / 4 \mathrm{~h}$, où $h$ est le rayon de la pupille de sortie, d'où un angle $\alpha_{i}$ de quelques minutes d'arc seulement. Deux solutions sont adoptées, soit l'association d'un seul interféromètre, ou prisme principal, de dédoublement moyen adapté à tous les objectifs, à une série de prismes auxiliaires (un par objectif) montés en tourelle dans le plan de la pupille du condenseur, soit que chaque objectif dispose de son propre interféromètre complété d'un prisme compensateur unique.

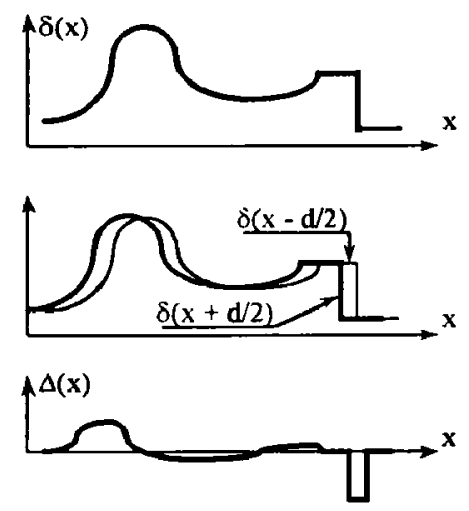

Figure 24 : Interféromètrie à faible dédoublement latéral ou contraste interférentiel

\subsubsection{L'interférométrie à modulation de phase}

Si l'interférométrie permet, outre la visualisation des objets de phase, de mesurer les déphasages qu'ils introduisent, c'est-à-dire de déterminer leur géométrie, leur réfractométrie, et ainsi de caractériser leurs constituants ou leurs réactions à des excitations spécifiques, cette mesure reste souvent difficile. Le déphasage local $\phi(x, y)$ est en effet mis en évidence par un éclairement dans le plan de l'image

$$
E(x, y)=\sin ^{2}[\phi(x, y) / 2]
$$

et le mesurer, et par là même différencier avances et retards de phase, impose de lui ajouter une phase compensatrice $\phi_{c}=-\phi(x, y)$ telle que

$$
E_{c}=\sin ^{2}\left\{\left[\phi(x, y)+\phi_{c}\right] / 2\right\}=0
$$

Cette détection d'éclairement minimal, effectuée par l'œil ou par un récepteur photoélectrique, est longue et difficile car relativement peu sensible,

$$
\mathrm{dE} / \mathrm{d} \psi=(\sin \psi) / 2
$$

tendant vers zéro (fig. 25) lorsque la phase compensée $\psi=\phi(x, y)+\phi_{\mathcal{C}}$ tend vers zéro. 
Une grande amélioration est obtenue par interférométrie à modulation de phase qui, associée à la microscopie, connait quelques développements ponctuels, surtout en laboratoire. Moduler la phase revient à ajouter au déphasage introduit par l'objet une phase $\phi_{A}$ variant périodiquement dans le temps à la fréquence $v_{0}$ avec une amplitude $\phi_{0}$, soit

$$
\phi_{\mathrm{A}}=\phi_{0} \sin 2 \pi v_{0} t
$$

et dans ces conditions, à l'instant $t$, l'éclairement, compte tenu de la présence du compensateur, est de la forme

$$
E(x, y, t)=\sin ^{2}\left[\left(\psi+\phi_{A}\right) / 2\right]=\left[1-\cos \left(\Psi+\phi_{0} \sin 2 \pi v_{0} t\right)\right] / 2
$$

et si le flux lumineux transmis par un diaphragme isoiant le point considéré est reçu par un récepteur photoélectrique, ce dernier fournit un signal $S(t)$ de la même forme, de fréquence fondamentale $v_{0}$, l'amplitude de sa composante à cette fréquence étant $J_{I}\left(\phi_{0}{ }^{\prime} \sin \psi\right.$ où $J_{I}$ est la fonction de Bessel de première espèce d'ordre 1.

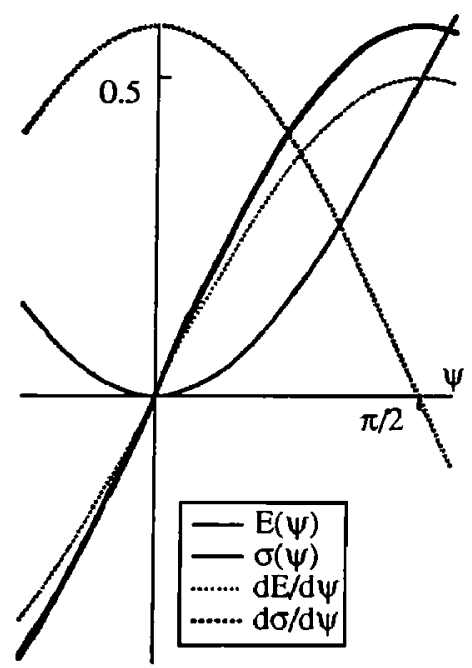

Figure 25 : Comparaison des performances des interférométries avec et sans modulation de phase

Par démodulation synchrone de ce signal à cette fréquence, utilisant un signal de référence délivré par le générateur produisant la modulation de phase, on obtient en sortie du détecteur synchrone un signal continu égal à l'amplitude de sa composante fondamentale

$$
\sigma=\mathbf{J}_{1}\left(\phi_{0}\right) \sin \psi
$$

On voit tous les avantages de cette méthode. Ce signal porte information sur le signe de $\psi$, c'est-à-dire qu'en absence de compensation, il est possible de différencier retards et avances de phase. La mesure se fait encore à l'aide du compensateur de telle sorte que l'on annule $\psi$, c'est-à-dire le signal d'erreur $\sigma$. Elle est plus sensible qu'en absence de modulation de phase, 
car le signal, pratiquement linéaire en fonction de $\psi$ lorsqu'il tend vers zéro (fig. 25), s'annule en changeant de signe sans passer par un minimum. La sensibilité

$$
\mathrm{d} \sigma / \mathrm{d} \psi=\mathrm{J}_{1}\left(\phi_{0}\right) \cos \psi
$$

tend, quand $\psi$ tend vers zéro, vers une constante $J_{I}\left(\phi_{0}\right)$ pouvant être optimisée par le choix de $\phi_{0}$ la rendant maximale. Enfin, ce signal d'erreur peut être utilisé pour piloter le déplacement du compensateur. Lorsque la valeur de compensation est atteinte, le signal d'erreur s'annule, entraînant l'arrêt du compensateur dans une position qu'il suffit d'enregistrer en fonction des coordonnées spatiales du point de l'objet.

L'interférométrie différentielle à ondes polarisées est particulièrement bien adaptée pour exploiter cette possibilité. Fournissant deux ondes polarisées perpendiculairement, au déphasage entre ces deux ondes introduit par l'objet peut s'ajouter un déphasage altematif produit par modulation d'un effet linéaire électro-optique (effet Pockels dans les cristaux), magnéto-optique (effet Faraday) ou photoélastique, résultant de la modulation du champ électrique, ou de l'induction magnétique, appliqué. L'installation de ces dispositifs par les constructeurs n'est pas systematique et est le plus souvent le fait des utilisateurs.

Comment moduler la phase?

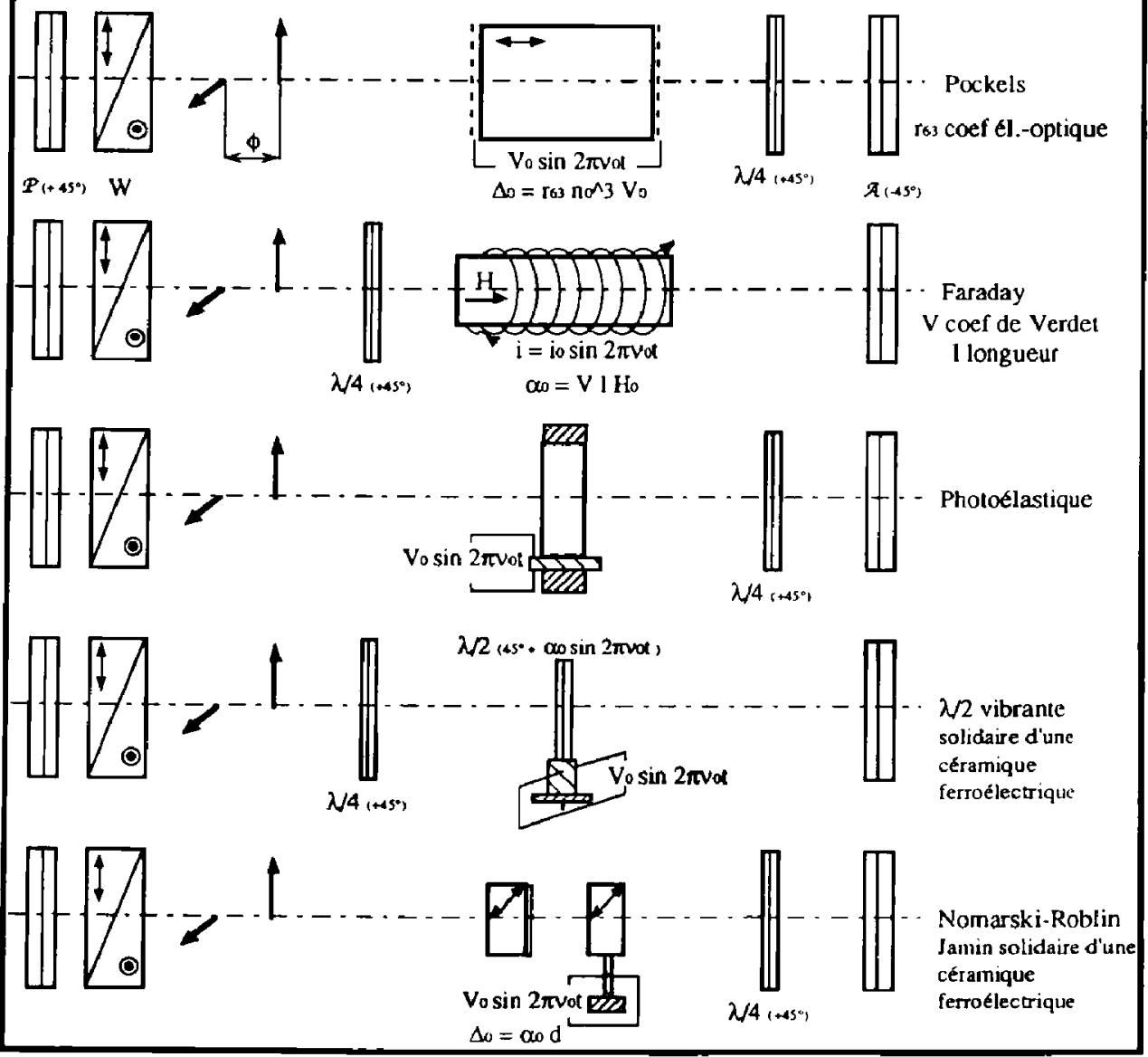




\section{LA MICROSCOPIE OPTIQUE À BALAYAGE}

\section{I Présentation de la méthode}

Contrairement à la microscopie traditionnelle où le champ est uniformément éclairé, en microscopie optique à balayage celui-ci est balayé de manière continue par l'image d'un point lumineux fournie par l'objectif lui-même, donc par un "spot" dont la dimension est du même ordre que la limite de résolution. Le flux lumineux transmis ou réfléchi en chaque point de l'objet, donc à un instant donné, est recueilli par un photorécepteur unique fournissant un signal portant information sur le niveau photométrique au point considéré, alors qu'en microscopie traditionnelle correspond à chaque point du champ un élément photosensible du récepteur (rétine de l'cil, émulsion photographique, tube de télévision, mosaique CCD), chacun d'eux pouvant être de sensibilité différente à une même excitation, voire de dimensions différentes, donc fournissant une résolution différente, si le récepteur est constitué d'éléments discrets.

La microscopie flying-spot est à l'origine de cette procédure. L'image du spot d'un tube à rayons cathodiques, ou tube analyseur, balayé dans deux directions est projetée dans le champ par le microscope complet (fig. 26). L'information sur le facteur de transmission en chaque point de l'objet est contenue dans le signal photoélectrique fourni par un photorécepteur placé dans la pupille du condenseur. Une image peut alors être affichée en utilisant ce signal pour moduler l'intensité du faisceau d'électrons $(Z)$ d'un tube moniteur à mémoire à entretien d'image à balayage synçhone de celui du tube analyseur. On peut encore ajouter ce signal, et à l'origine ce fut probablement l'usage le plus fréquent en microscopie flying-spot, au signal de balayage image $(Y)$ du moniteur fonctionnant ainsi en tube oscilloscope et afficher pour chaque ligne une courbe représentative de ses variations photométriques. Les deux types d'information, par application du signal photoélectrique tant en $Z$ qu'en $Y$, peuvent également être affichées simultanément.

Avec ce mode d'éclairage, mise à part l'énergie résiduelle dans les pieds de la tache de diffraction, rien ne vient éclairer les autres points du champ qui ne diffusent donc pas de lumière parasite constituant un fond lumineux tendant à diminuer le contraste de l'image. Ce contraste peut être modifié et ses faibles valeurs peuvent être augmentées en jouant sur le gain d'un amplificateur, en accumulant les signaux pendant plusieurs balayages, ou, ce à quoi se prêtait bien la méthode flying-spot, en adaptant par contre-réaction la luminosité du spot à la transparence de l'objet. Cette méthode, dans la mesure où elle utilisait des tubes à déflexion électrostatique, offrait l'avantage d'une grande souplesse de gamme de fréquences de balayage possible permettant leur adaptation aux caractéristiques d'un objet tant spatiales que temporelles. Par contre ce type de réalisation présentait les inconvénients d'utiliser un spot inhomogène dans le champ en dimension et intensité, de ne pas éliminer toute lumière parasite à cause de la rémanence du phosphore de l'analyseur, d'être faiblement lumineux, la pupille du microscope ne prélevant qu'une très faible part de la lumière émise, enfin, à l'époque de son développement, d'être difficile à mettre en cuvre. En fait l'intérêt premier du balayage, en l'absence de récepteurs à balayage électronique (tube TV, dissecteur d'image), a certainement été de permettre la microscopie quantitative, le signal photoélectrique n'étant porteur à chaque instant que de l'information relative à un seul point résolu dans le champ. En effet, l'observation de l'image ne paraît pas toujours suffisante et il est souhaitable de quantifier l'information qu'elle contient (aujourd'hui le plus souvent en la numérisant), c'est-à-dire d'affecter à chacun des points du champ analysé, repéré par sa position sur une ligne et le rang 
de cette dernière, un nombre représentatif de son niveau photométrique. Le flying-spot était tout à fait adapté à cette métrologie, le tube moniteur pouvant, comme nous l'avons évoqué, effectuer l'oscilloscopie du signal délivré et il permit les toutes premières applications en analyse d'image ou microscopie quantitative.

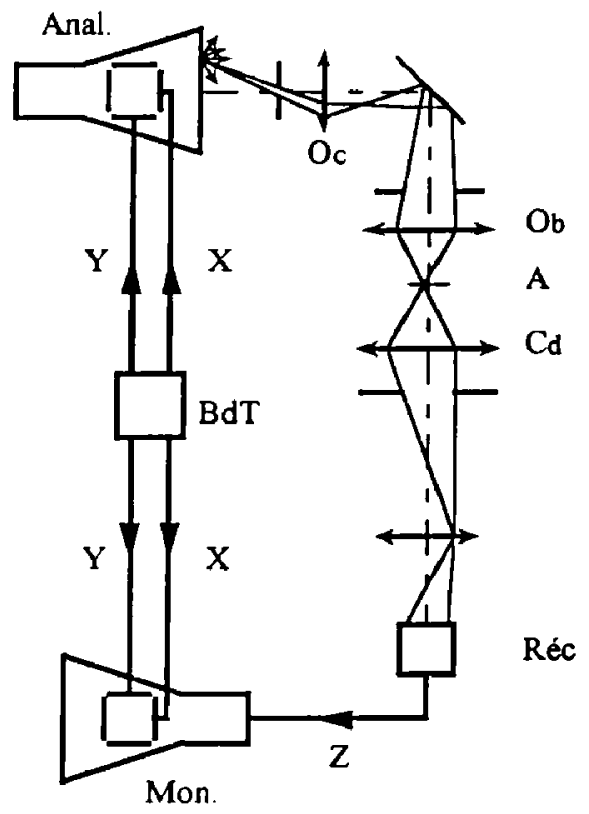

Figure 26 : Schéma de la microscopie flying-spot

Ses inconvénients masquant, au point de les oublier, ses avantages, cette méthode fut détrônée au profit du balayage électronique de l'image permis par le développement et les progrès des tubes récepteurs de télévision, tout d'abord pour faciliter la visualisation collective par plusieurs observateurs, plus récemment pour atteindre la numérisation. Cette technique et son analogue basée sur l'emploi d'une barrette de récepteurs couplée à un déplacement perpendiculaire de l'objet ou plus généralement d'une mosaique de récepteurs, si elle tend à s'améliorer, présente néanmoins encore des inconvénients rejoignant d'ailleurs ceux du procédé flying-spot. La limitation du nombre de points d'analyse, en tout état de cause inférieur au nombre de points résolus dans le champ de l'objectif du microscope (pouvant atteindre 2500 sur un diamètre), conduit soit à celle du format, soit à une perte de résolution, et bien souvent au choix d'un compromis entre ces deux défauts de qualité. Les variations de sensibilité entre les éléments d'un récepteur multiple, ou de la surface photosensible d'un tube récepteur de télévision, d'effet analogue aux variations de luminance du spot du tube à rayons cathodiques, perturbent la linéarité de la réponse. De plus la fréquence de balayage reste pratiquement imposée soit à une valeur élevée dans le cas de la télévision pure, soit à une valeur relativement faible quand l'objet doit être déplacé dans une direction, à l'encontre de la gamme étendue que 
permettait le flying-spot. Par contre, les possibilités actuelles ou attendues des moyens informatiques, la tendance des constructeurs, à la demande des utilisateurs, à produire des objectifs de microscope à champ plan toujours plus grand, militent en faveur d'un traitement de l'information pour l'ensemble des points résolus de ce champ (ce qui est encore loin d'être atteint). L'illuminer par l'image d'un point lumineux, de dimension conforme à la résolution de l'objectif revient alors naturellement en faveur, sans compter que son excitation par une radiation lumineuse spécifique peut être à l'origine d'effets porteurs d'information. On a vu alors deux tendances se dessiner au niveau des recherches, qui au stade du développement, compte tenu de leurs intérêts respectifs, se sont pratiquement réunies.

La première de ces tendances, présentant de nouveaux avantages que nous préciserons au paragraphe suivant, adoptant une configuration dite confocale, consiste en l'emploi de deux objectifs identiques conjuguant (fig. 27 a) par l'intermédiaire du plan objet $A$ une source $S$ et un récepteur $R$ tous deux ponctuels, c'est-à-dire de structures dans le plan objet analogues à celle de la tache de diffraction commune aux deux objectifs. L'objet est animé d'un double mouvement de translations orthogonales par rapport à l'ensemble des deux microscopes. La seconde tendance, plus proche du systeme flying-spot, utilise un faisceau lumineux issu d'un laser focalisé dans le plan de l'objet à travers le microscope (fig. $27 \mathrm{~b}$ ). Ce faisceau étant défléchi dans deux directions orthogonales autour du centre de la pupille, le flux lumineux transmis est recueilli par un photorécepteur unique $R$ placé dans la pupille du condenseur $C_{d}$. Cette méthode de balayage laser offre pratiquement l'avantage d'applications liées à l'irradiation ponctuelle par un faisceau laser intense.

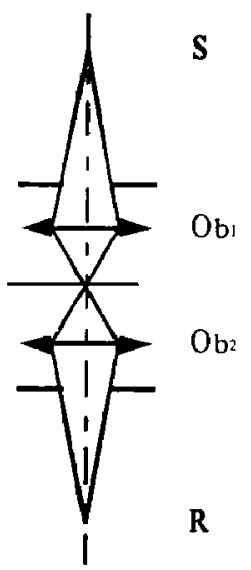

(a)

$\mathbf{R}$

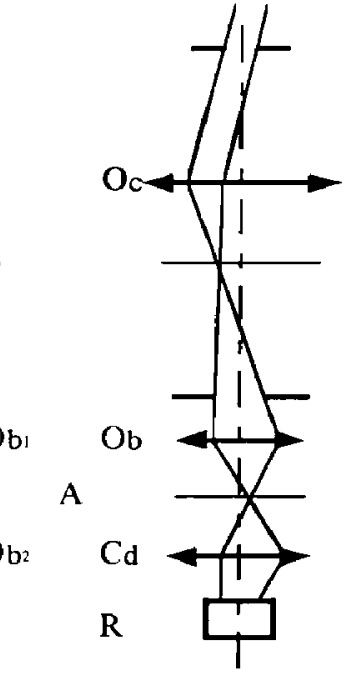

(b)

Figure 27 : Microscopies confocale (a) et à balayage laser (b) 


\subsection{La microscopie confocale et ses avantages}

Les avantages mis en évidence récemment de la confocalité, loin d'être négligeables, sont à l'origine de nouvelles potentialités en microscopie optique. Projeter un point lumineux dans le plan objet revient à l'éclairer localement suivant une répartition identique à la tache de diffraction de l'objectif :

$$
S(\rho)=\left[2 J_{1}(Z) / Z\right]^{2}
$$

où $Z=(2 \pi \rho n \sin U) / \lambda$. Capter cette énergie par un récepteur également ponctuel par l'intermédiaire d'un objectif identique, revient à l'analyser dans ce même plan objet avec une sensibilité variant spatialement selon la même loi :

$$
R(\rho)=\left[2 J_{1}(Z) / Z\right]^{2}
$$

Ainsi, globalement, tout se passe comme si le "point résolu" du champ avait la structure beaucoup plus fine du carré d'une tache de diffraction (fig. 28) :

$$
E_{c}(\rho)=S(\rho) R(\rho)=\left[2 J_{1}(Z) / Z\right]^{4}
$$

L'objet n'étant éclairé instantanément qu'en un seul point, plutôt que la limite de résolution, semble-t-il plus logique de considérer la classique limite de séparation entre deux points définie en adoptant le critère selon lequel deux points sont séparés, en éclairage incohérent, si leur distance est égale au rayon de la tache de diffraction, ce qui entraine dans l'image globale des deux points une baisse de contraste de $25 \%$, soit $\rho_{i}=1,22 \lambda / 2 \mathrm{n} \sin \mathrm{U}$.

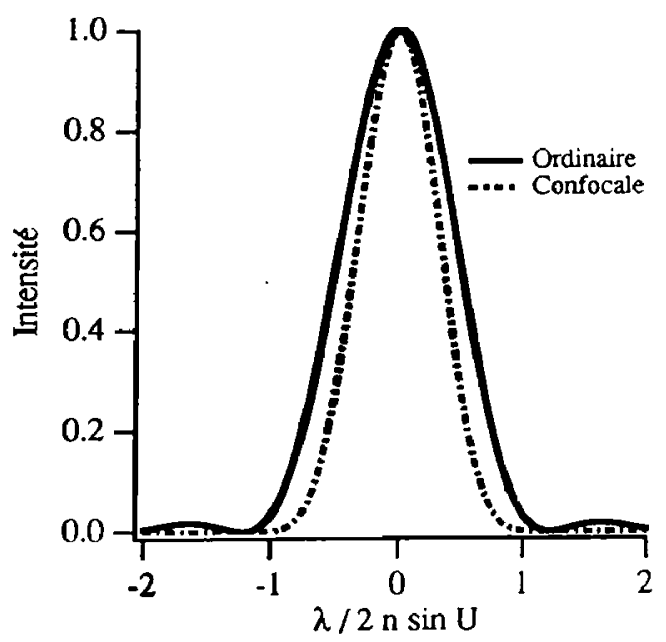

Figure 28 : Comparaison des "images" d'un point en microscopies ordinaire et confocale 
En adoptant ce même critère de baisse de contraste entre deux points de même transmission balayés successivement en microscopie confocale, cette limite est nettement améliorée puisqu'elle correspond (fig. 29) à une distance entre deux points réduite à

$$
\rho_{\mathrm{C}}=0,875 \lambda / 2 \mathrm{n} \sin \mathrm{U}
$$

soit une limite au moins 1,4 fois meilleure car en fait on peut admettre que le récepteur photoélectrique présente une meilleure sensibilité au contraste que l'œil.

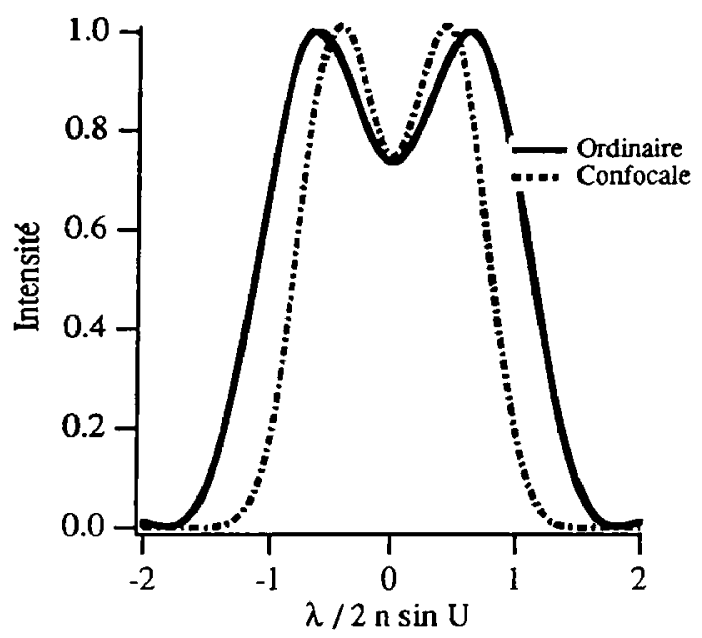

Figure 29 : Comparaison des "limites de séparation" entre deux points en microscopies ordinaire et confocale

Si le concept de limite de résolution, qui fait appel à la notion de fréquence spatiale, est difficile à retenir puisqu'il se rapporte à un objet étendu, de répartition spatiale périodique en amplitude ou en intensité, diffractant la lumière dans toute l'ouverture de l'instrument, il est néanmoins possible de l'appréhender. En calculant par intégration la modulation du signal temporel, c'est-à-dire la structure de l'image, lorsqu'un tel spot $E_{c}(\rho)$ balaie une structure périodique en intensité, on montre que pour une période égale à la limite de résolution absolue $\lambda / 2 n \sin U$ définie précédemment, le contraste est encore de $16 \%$ au lieu d'être nul alors qu'il devient négligeable pour une période 1,4 fois plus faible (fig. 30). Il y a donc une amélioration sensible du facteur de modulation pour les fréquences spatiales voisines de la fréquence de coupure classique rencontrée en microscopie ordinaire.

À cet avantage, s'ajoute celui de l'amélioration du rapport signal à bruit dû au fait que les autres points du champ ne sont pas éclairés et ne peuvent donc diffuser de lumière. Il faut bien comprendre qu'il ne s'agit pas seulement des points d'un même plan de mise au point, mais que cet effet en microscopie ordinaire est produit par tout point de l'épaisseur de la préparation. L'effet n'est donc pas totalement supprimé pour les points de l'objet épais observé situés (fig. 31) à l'intérieur du cône d'éclairage d'ouverture U. Il est néanmoins notablement diminué pour deux raisons 


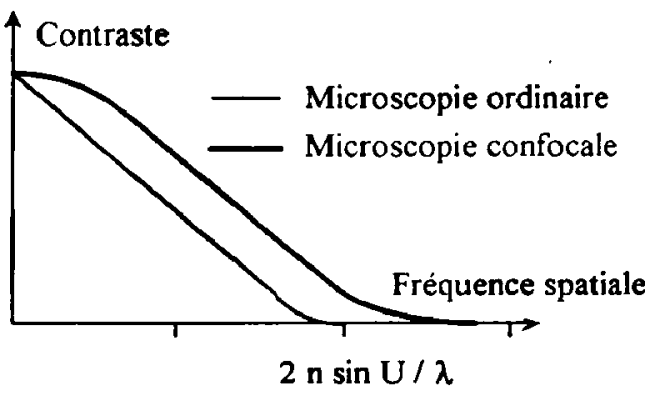

Figure 30 : Comparaison des limites de résolution en microscopies ordinaire et confocale

La première est qu'il n'existe pas physiquement de source et de récepteur ponctuels. Matériellement, pour les réaliser, on les limite en plaçant dans leur plan un petit trou devant avoir un diamètre égal à une fraction de celui du premier anneau de la tache de diffraction (de l'ordre de $1 / 5$ à $1 / 10$ est suffisant, soit des trous, pour une fraction $1 / k=1 / 5$, de diamètre 5 à $10 \mu \mathrm{m}$ suivant que l'objectif est faible ou fort) afin que leur image géométrique dans le plan de l'objet soit assimilable à un point lumineux. $\mathrm{Ce}$ diaphragme a pour effet de limiter très fortement l'étendue géométrique en dehors du plan de mise au point $A$, donc l'énergie rayonnée relative aux points objets situés à l'extérieur des plans $C C$ distants de $A$ de

$$
z_{0}= \pm \rho_{i} / k n \sin U= \pm 1,22 \lambda / 2 k n^{2} \sin ^{2} U
$$

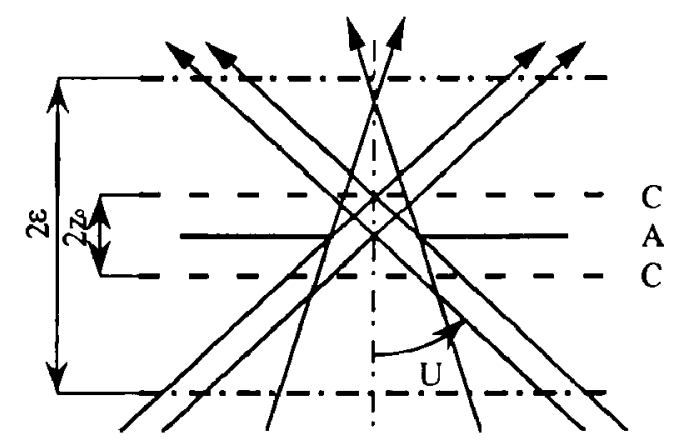

Figure 31 : Justification de la diminution de la profondeur de champ en microscopie confocale

La seconde raison est que lorsqu'une pupille diffracte l'onde émise par un point lumineux (source cohérente), l'énergie transportée dans la direction de l'axe optique varie suivant la loi

$$
\mathrm{S}(\mathrm{z})=\{[\sin (\pi \Delta / \lambda)] /(\pi \Delta / \lambda)\}^{2}
$$

où $\Delta$ est l'écart aberrant de l'onde convergeant dans un plan de défaut de mise au point $z$ par rapport à une surface d'onde sphérique théorique:

$$
\Delta=\mathrm{nz}(1-\cos \mathrm{U})
$$


Par analogie, on peut considérer que le long de l'axe $z$, la sensibilité $R(z)$ du récepteur varie dans les mêmes proportions et qu'ainsi l'éclairement en un point de l'axe est de la forme

$$
\mathrm{E}(\mathrm{z})=\mathrm{S}(\mathrm{z}) \mathrm{R}(\mathrm{z})=[(\sin \mathrm{Kz}) / \mathrm{Kz}]^{4}
$$

où $K=n \pi(I-\cos U) / \lambda$. Cette loi de variation, rapidement décroissante, contribue fortement à l'amélioration du rapport signal à bruit et pour ces deux raisons, la microscopie confocale présente sur la microscopie ordinaire un avantage indéniable. En effet, pour cette dernière, nous admettons que tout défaut de mise au point n'entraînant pas d'écart aberrant supérieur à $\lambda / 4$ est sans influence détectable sur la qualité de l'image, ce qui définit en fait une profondeur de champ limitée aux plans $M M$ distants de $A$ de

$$
\varepsilon= \pm \lambda / 4 n(1-\cos U)
$$

En microscopie confocale par contre, pour cette valeur de $\varepsilon, E(z)$ est déjà diminué d'un tiers et pour des trous source et récepteur de diamètre égal au cinquième de celui de la tache de diffraction, l'étendue géométrique est réduite dans un rapport de l'ordre de 20 . On peut donc admettre une profondeur de champ, c'est-à-dire une résolution longitudinale, de l'ordre de $z_{o}$ comprise entre 1 (objectif $10 / 0,25$ ) et $0,04 \mu \mathrm{m}$ (objectif $100 / 1,25$ ), donc 4 à 5 fois plus faible qu'en microscopie ordinaire. Cette possibilité d'analyser un objet suivant des coupes beaucoup plus fines pour des plans de mise au point plus rapprochés permettant sa reconstruction en trois dimensions est l'avantage essentiel de cette microscopie.

\subsection{La conception instrumentale}

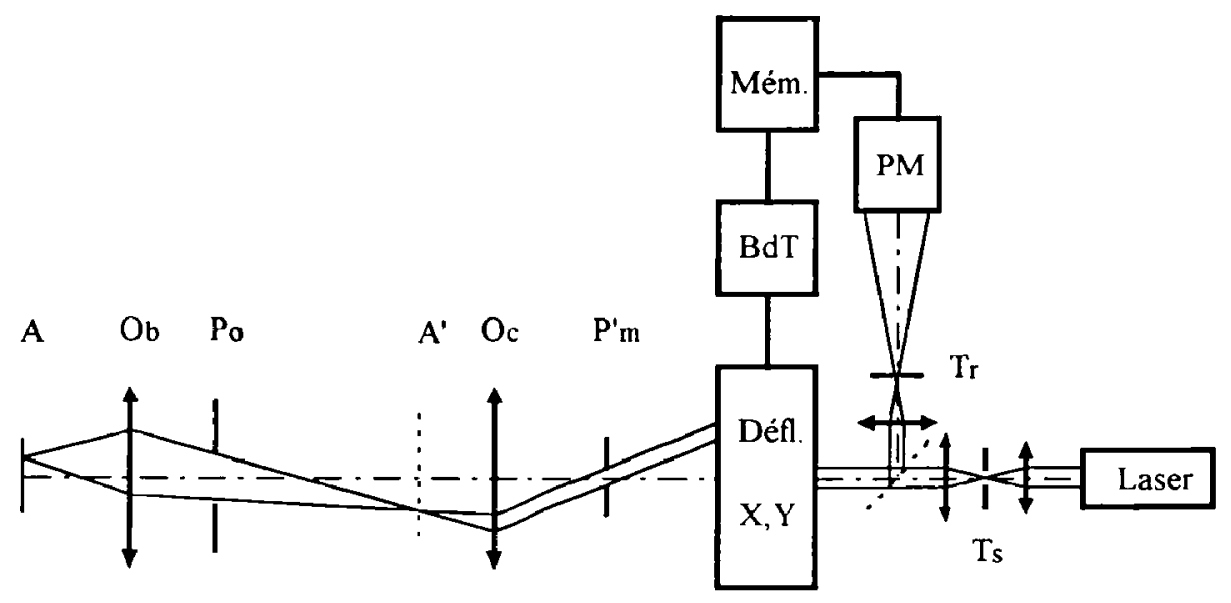

Figure 32 : Schéma de la microscopic confocale à balayage laser

La conception d'un instrument conforme à la figure 27 a est particulièrement difficile en lumière transmise car elle implique que deux corps de microscope identiques soient centrés avec une incertitude inférieure d'un ordre de grandeur à leur tache de diffraction commune et que leurs plans objets restent toujours simultanément confondus. D'autre part, dans un tel 
projet, le balayage ne peut se faire que par double déplacement de l'ensemble des deux corps de microscope, ou de la platine, ensembles mécaniques encombrants et lourds, ce qui en limite nécessairement la fréquence et nuit à la stabilité de l'instrument. Il n'en est pas de même en lumière réfléchie où, un seul microscope étant utilisé, il est plus aisé d'assurer la conjugaison de deux trous de dimensions raisonnables dans l'espace intermédiaire (fig. 32). Sous réserve de faire retraverser le double système déflecteur par la lumière réfléchie afin de la ramener dans une direction fixe, il est alors possible en microscopie confocale par réflexion de ne pas procéder au balayage de l'objet par son propre déplacement, mais d'utiliser la double déflexion d'un faisceau laser qui peut s'effectuer dans une large gamme de fréquences pouvant atteindre de grandes valeurs. C'est sur ce schéma, qui impose un besoin supplémentaire d'éléments optiques, que sont aujourd'hui construits la plupart des instruments existants.

Le système déflecteur est composé de deux éléments assurant respectivement les balayages $X$ ou ligne et $Y$ ou image. Ces deux composantes doivent être simultanément conjuguées d'un plan pupillaire. Les conditions requises sont qu'ils n'affectent pas la résolution du microscope, qu'ils permettent de couvrir l'ensemble de son champ (ce qui n'est en général pas encore réalisé car trop exigeant en capacité informatique, incompatible avec les possibilités des systèmes de visualisation, ou plus simplement en contradiction avec la conservation de la résolution), qu'ils n'interdisent pas l'emploi alternatif ou simultané en microscopie traditionnelle en lumière transmise ou réfléchie. Plusieurs types de dispositifs sont disponibles et peuvent être utilisés seuls ou en association pour assurer les deux balayages.

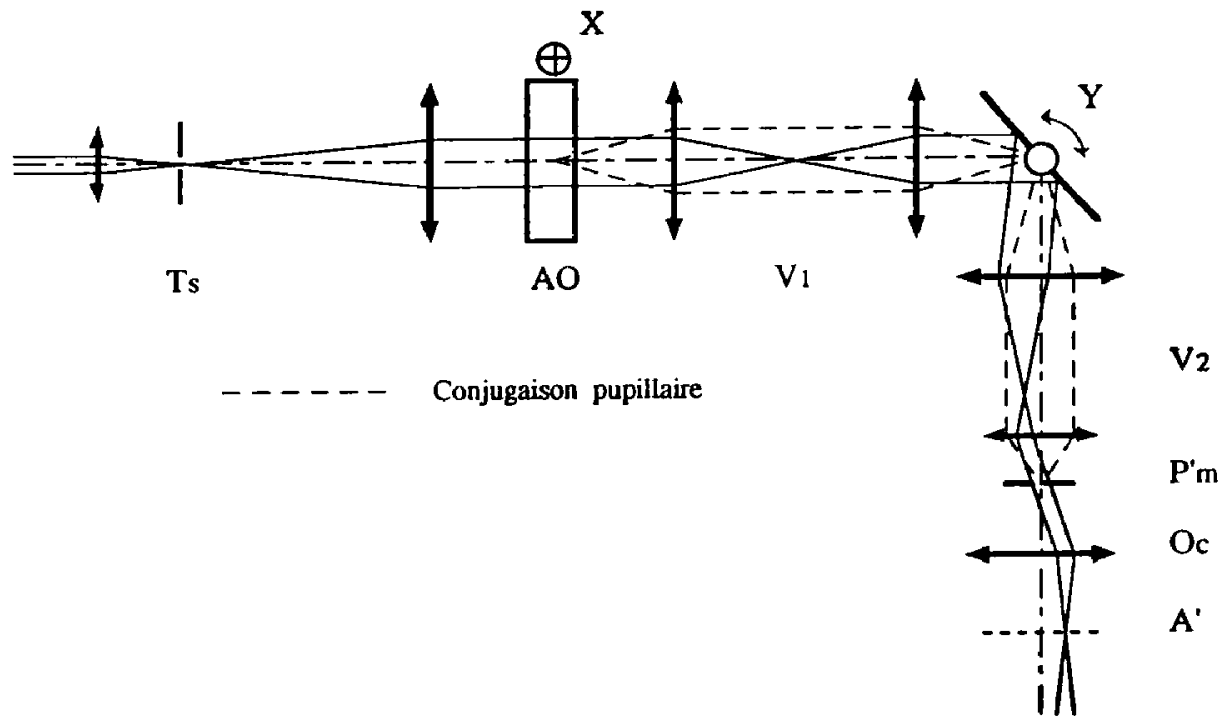

Figure 33 : Schéma adoptant l'emploi d'un déflocteur acousto-optique pour assurer le balayage "ligne"

L'utilisation (fig. 33) d'un déflecteur acousto-optique linéaire $A O$ au moins pour le balayage ligne permet une fréquence variable atteignant une valeur de l'ordre de celle de la télévision (environ $60 \mu \mathrm{s}$ par ligne), un repérage répétitif des points sans dépasser une résolution d'environ 450 points par ligne, suffisante si l'on se limite à une trame carrée de $512 \times 512$ pixels. Outre un défaut d'encombrement, ce système impose, si l'on ne veut pas 
perdre trop d'énergie lumineuse, l'emploi d'une optique cylindrique destinée à élargir le faisceau au niveau du cristal actif dans la seule direction du reseau induit pour augmenter la zone d'interaction phonon-photon et faire que la dispersion par le réseau soit effective. Ceci rend difficile, par la suite, d'assurer la double conjugaison pupillaire et objective et la correction parfaite de l'astigmatisme introduit. Un second défaut provient du fait que la déflexion fournie par le réseau optique induit dans le cristal est produite sous un angle dépendant du pas variable de ce réseau alors que son inclinaison par rapport au faisceau incident reste fixe. Dans ces conditions, l'efficacité du réseau varie et la perte d'énergie transmise dans l'ordre diffracté utile peut atteindre $50 \%$ pour la déflexion maximale. L'éclairement dans le plan objet n'est donc pas constant, ce qui impose une correction au niveau du traitement de signal et interdit la quantification des effets d'interaction lumièrematière au niveau de l'objet. Si la lumière de retour est de longueur d'onde différente (en fluorescence par exemple), elle est défléchie à la traversée du système et revient donc finalement dans une direction non fixe dépendant de la longueur d'onde, ce qui est un inconvénient suffisamment majeur pour interdire l'emploi de ce système pour l'application considérée.

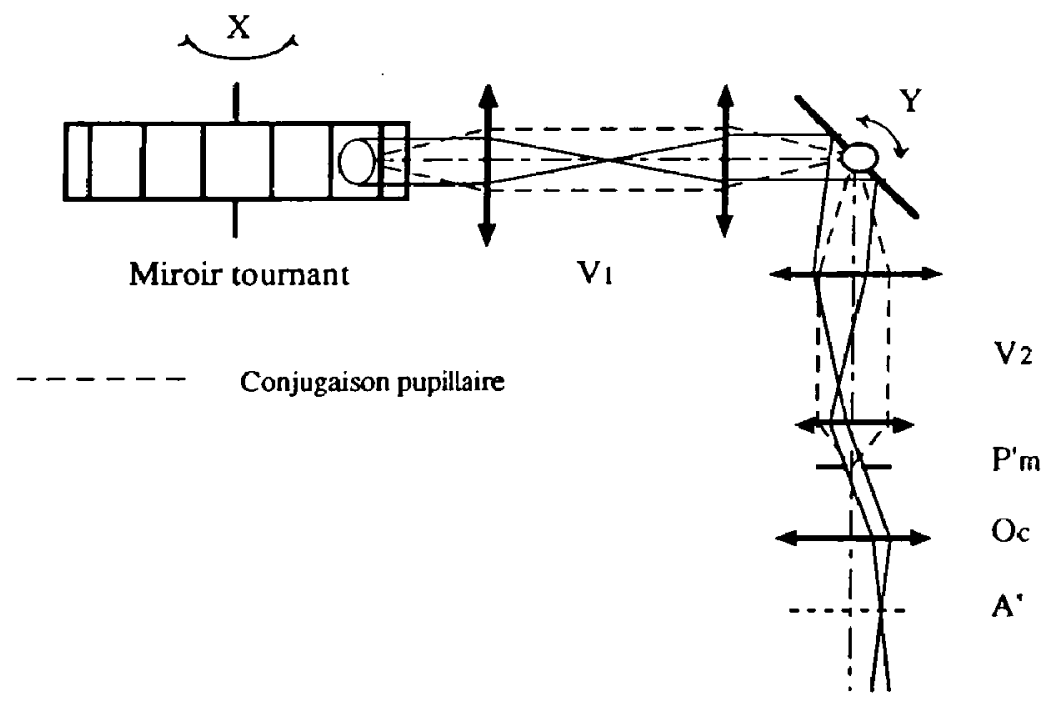

Figure 34 : Schéma adoptant l'emploi d'un miroir tournant multiface pour assurer le balayage "ligne"

L'emploi (fig. 34) d'un miroir tournamt mulliface, permet d'obtenir une fréquence ligne assez grande, mais une fréquence image limitée du fait des temps morts causés par une trop grande déflexion. Il présente un premier inconvénient du fait que la position du centre instantané de rotation du faisceau lumineux, qui doit être dans la pupille, varie avec l'angle de déflexion, ce qui crée une aberration: pupillaire parfois sensible. Pratiquement, l'inconvénient le plus grave provient des défauts de pyramidité propres à chacune des facettes conduisant à limiter le nombre de lignes afin qu'elles ne soient pas jointives pour ne pas risquer qu'elles se coupent. Il est bien sûr possible d'y remédier au prix d'un composant coûteux, éventuellement à facettes individuellement réglables, ce qui, augmentant l'encombrement et le poids, risque d'être source de vibrations. 
La solution la plus généralement adoptée (fig. 35) est l'utilisation de miroirs solidaires d'équipages galvanométriques sous deux formes distinctes. Les galvanomètres résonants, employés pour le balayage ligne à $8 \mathrm{kHz}$ par exemple, permettent d'atteindre pour une image de 512 lignes de 512 points la fréquence cinéma ( 16 images par seconde). Ce balayage, dont la fréquence ne peut être modifiée, n'est pas linéaire, ce qui implique que son générateur doit piloter l'ensemble des autres composants électroniques du montage et de longs temps morts entre lignes pour éliminer les portions à vitesse trop lente (elle s'annule en extrémité de ligne). Cette non-linéarité entraîne une variation de résolution si les points sont définis par une horloge régulière assurant des temps de vol du spot constants, donc des luminations constantes, ou au contraire une variation de l'excitation lumineuse si l'horloge varie sinusoïdalement pour conserver même dimension spatiale aux points analysés. Les galvanomètres asservis au contraire permettent un balayage linéaire avec une fréquence de l'ordre de $250 \mathrm{~Hz}$ au mieux qui les rend aptes à être associés pour le balayage image à tous les systèmes qui viennent d'être évoqués. En balayage ligne, ils permettent un temps de vol de l'ordre de $8 \mu$ s en chaque point d'une ligne de 512 points, ce qui n'est pas incompatible avec les exigences d'excitation ou d'analyse de signal propres à certaines applications. Le temps de saisie d'une image de 512 lignes est alors de 2 secondes. Les temps morts (nécessaires à l'amortissement des rebonds en fin de chaque période) sont relativement beaucoup plus courts qu'avec les systèmes résonants.

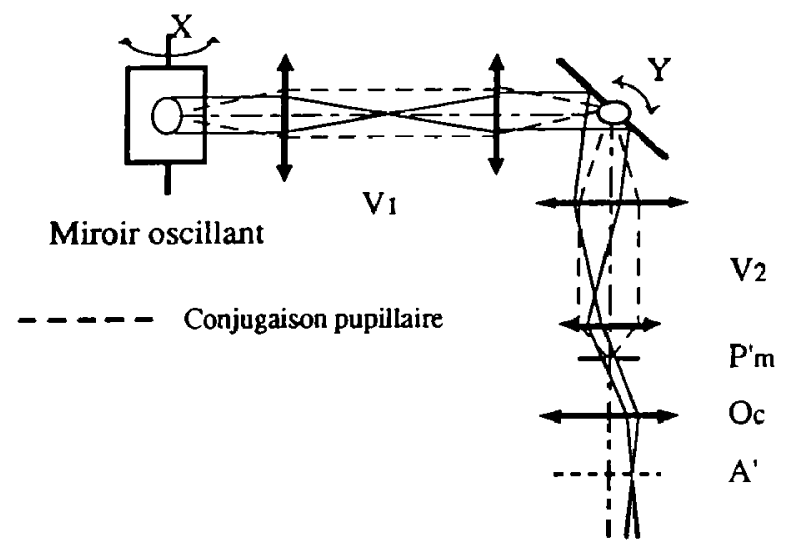

Figure 35 : Schéma adoptant l'emploi de miroirs oscillants pour assurer les balayages "ligne" et "image"

La source de lumière utilisée est un laser à gaz peu divergent émettant un faisceau monochromatique de faible puissance (de l'ordre du milliwatt) suffisante pour que sa focalisation dans le plan de l'objet crée un éclairement énergétique s'avérant souvent trop intense et qu'il faudra donc atténuer. Dans la zone du spectre visible pour laquelle les optiques sont couramment corrigées, on utilise les lasers à gaz à émission continue HeCd (442 nm), $\mathrm{Ar}^{+}$(9 raies entre 454 et $\left.514 \mathrm{~nm}\right), \mathrm{HeNe}(633$ et plus rarement 543 et $594 \mathrm{~nm})$. Dans la mesure où l'on dispose d'une optique le permettant, l'extension est possible vers le domaine du proche ultraviolet avec le laser HeCd $(325 \mathrm{~nm})$, notamment pour exciter la fluorescence, ce que permettent également sa raie bleue et les raies les plus courtes de l'argon ionisé, ou vers le proche infrarouge avec le laser HeNe $(1152 \mathrm{~nm})$. Cette lumière est successivement focalisée 
(fig. 32) sur le trou source $T_{S}$ conjugué du plan objet, collimatée avec un diamètre compatible avec celui de la pupille $P_{m}^{\prime}$ du microscope, défléchie angulairement dans deux directions perpendiculaires autour du centre de la pupille, puis après réflexion sur l'objet et retraversée du déflecteur, focalisée sur le trou récepteur $T_{r}$ conjugué du trou source, reçue par un photorécepteur $P M$.

Les objectifs utilisés sont ceux qui assurent classiquement la résolution souhaitée. Ils doivent être associés à un oculaire de caractéristiques telles que le diamètre de la pupille de sortie du microscope ainsi constitué soit compatible avec celui du faisceau laser dans cet espace, afin que la pupille de l'objectif soit effectivement couverte. Compte tenu de ce mode d'éclairage, l'emploi d'objectifs corrigés pour un champ plan (planachromats ou planapochromats) est nécessaire.

Le détecteur, si le balayage est lent et compte tenu de l'éclairement intense, n'est pas nécessairement sophistiqué. Néanmoins, si les flux lumineux sont faibles, par exemple en épifluorescence, ou pour l'observation en lumière réfléchie d'objets réputés transparents, et/ou si cette lumière est de bande spectrale large, l'emploi d'un photomultiplicateur s'impose. Servant seulement à recueillir un flux lumineux, il n'est pas nécessaire que sa surface photosensible soit placée dans un plan image, donc confondue avec le trou récepteur du système confocal, mais seulement suffisant que ses dimensions ne viennent pas limiter l'étendue du faisceau définie par ce trou et la pupille située dans le même espace.

L'instrument est associé à des éléments périphériques de caractéristiques compatibles avec celles du balayage, pilotés par ses générateurs, ou le pilotant eux-mêmes. Ces éléments assurent la numérisation du signal analogique du photorécepteur soit pour le traiter en temps réel, soit pour le stocker en vue d'un traitement ultérieur ou d'un transfert à une fréquence différente compatible avec celle d'un affichage.

Pour terminer cette partie instrumentale, nous évoquerons le microscope confocal par réflexion le plus anciennement connu (1968) permettant l'observation visuelle du champ. Muni (fig. 36) d'un disque tournant partagé en secteurs percés de multiples trous disposés le long de spirales d'Archimède (disque de Nipkow), à chaque instant les trous éclairés d'un secteur sont conjugués de ceux du secteur opposé. Le champ est entièrement couvert par un secteur et balayé autant de fois par tour que le nombre de secteurs. La vitesse de rotation est réglable pour obtenir l'impression d'une observation totale tant visuelle que photographique ou vidéo. Cette solution ne permet pas l'éclairage par un faisceau laser focalisé et met en œuvre une source et un système d'éclairage classique pour illuminer le champ. Moins adapté à la microscopie par fluorescence, il possède les autres avantages de la microscopie confocale.

\subsection{Les domaines d'application du microscope confocal}

Le signal photoèlectrique peut être utilisé pour l'affichage $Z$ de l'image résolue du champ objet ou de sa répartition photométrique $Y$ en temps réel ou être stocké en mémoire $M \dot{m}$ (fig. 37). Susceptible de tout traitement actif (modification de contraste, effacement de niveaux sélectionnés, discrimination de contours, ...) par contre-réaction $C R$ sur le niveau d'émission de la source, échantillonné par les impulsions d'une horloge $\mathrm{Cpt}$ dont le comptage fournit l'adresse des points résolus, il peut recevoir tout traitement passif après réception. Si un seul balayage suffit à saisir l'information pour un objet statique, le balayage continu ou d'images successives, sous réserve d'adapter sa fréquence à la vitesse d'évolution, donne accès à la dynamique d'un phénomène: mobilité d'une cellule, transfert d'une information, croissance ou décroissance d'un effet induit, ... Grâce à la très faible profondeur de champ de l'instrument, 
ces potentialités peuvent s'appliquer à un ensemble de plans d'analyse faiblement équidistants conduisant à leur étude en trois dimensions.

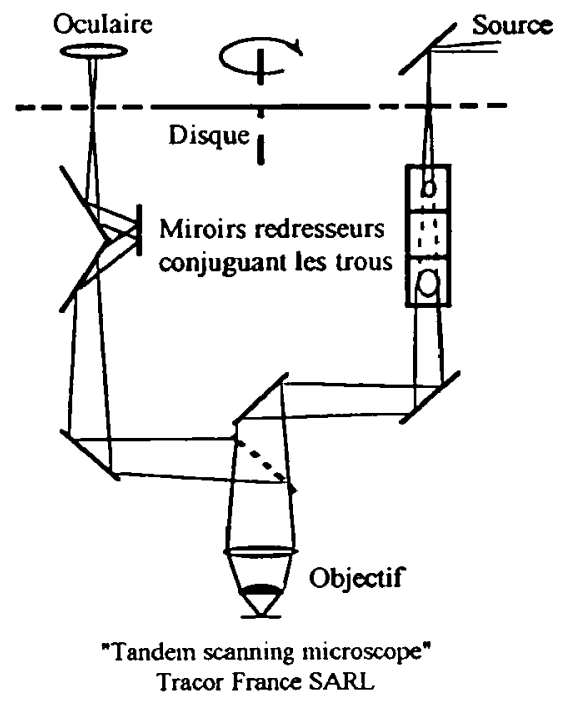

Figure 36 : Schéma du microscope confocal "visuel" proposé par Tracor

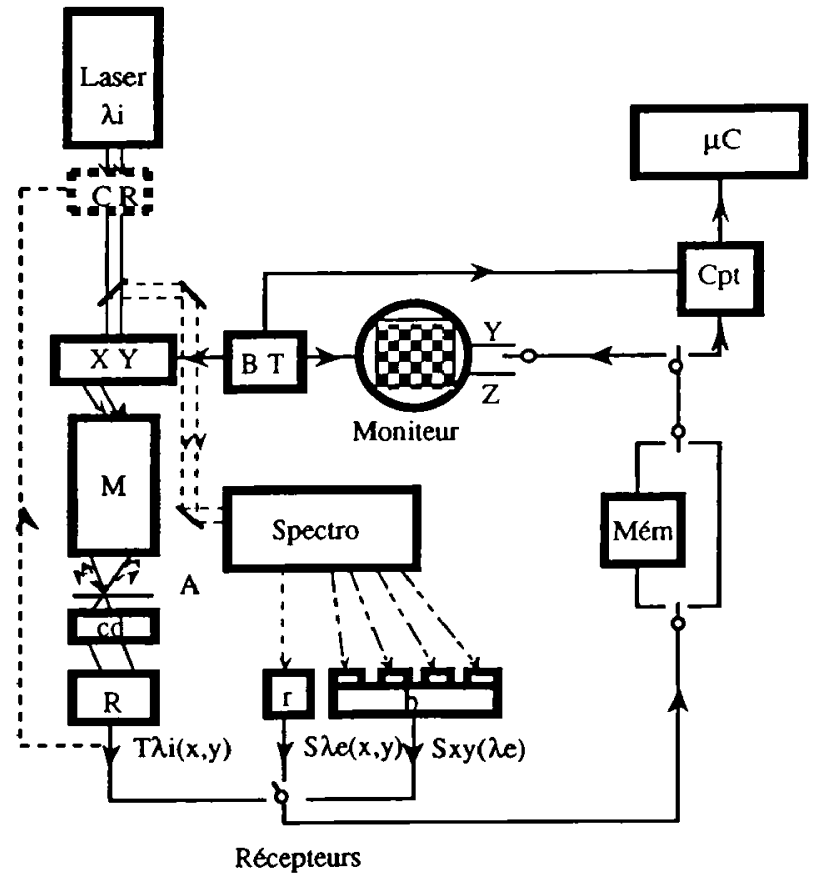

Figure 37 : Schéma représentatif des différents modes d'utilisation d'un microscope confocal 
L'irradiation monochromatique ponctuelle intense permet l'étude photométrique par l'accès au facteur de transmission $T_{\lambda j}(x, y)$ à longueur d'onde donnée, pour un ensemble de longueurs d'onde discrètes (avec plusieurs lasers ou un laser multiraie), une bande de faible largeur spectrale (avec un laser accordable). Dans le schéma confocal, la lumière réémise par fluorescence ou effet Raman étant ramenée dans une direction fixe après retraversée du modulateur $X Y$, peut être analysée spectralement en fonction des coordonnées de l'objet à longueur d'onde donnée $S_{\lambda e}(x, y)$ ou de la longueur d'onde en un point donné $S_{x y}\left(\lambda_{e}\right)$ en l'introduisant dans un spectromètre mono ou multicanal dont la fente d'entrée est conjuguée du trou source.

Ce type d'irradiation présente un avantage indéniable sur les méthodes d'excitation habituelles. En effet, s'il est possible d'adapter la longueur d'onde excitatrice à la spécificité du phénomène, le spectre de lumière réémise (fig. 38) est parfaitement caractéristique de cette longueur d'onde car il n'est pas l'effet de l'ensemble des radiations transmises par le filtre d'excitation habituellement associé à une source spectralement continue utilisée en microscopie ordinaire. D'autre part, le spectre analysé est bien caractéristique de la seule fluorescence car il ne s'y superpose pas les résidus de celui de la source transmis par le filtre d'arrêt au delà de sa fréquence de coupure, en quantité bien sûr faible, mais parfois non négligeable devant celle de la lumière réémise. Par ailleurs, certains phénomènes de fluorescence ou Raman sont si faibles qu'ils ne sont détectés en microscopie ordinaire qu'en prolongeant la durée d'excitation, ce qui masque par intégration toutes informations évolutives.
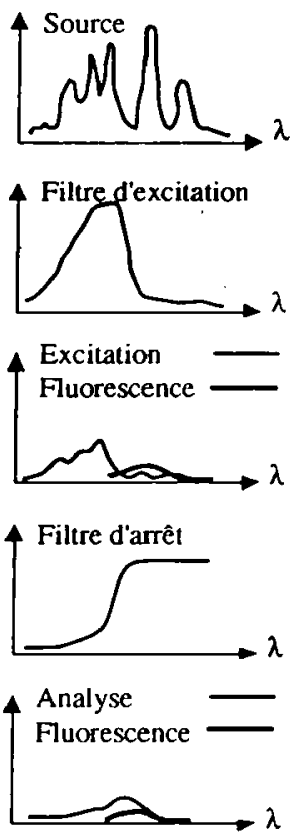

Méthode classique Microscopie ordinaire
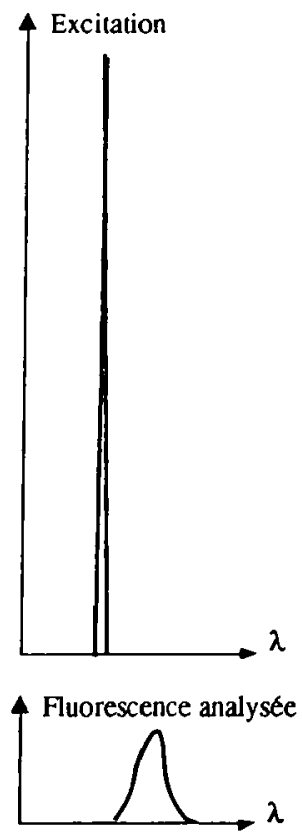

Excitation Laser Microscopie confocale

Figure 38 : Comparaison des performances obtenues en microscopies par fluorescence ordinaire et confocale 
L'irradiation ponctuelle laser permet d'augmenter notablement la densité spatiale de puissance énergétique qui suivant l'objectif utilisé atteint de 0,5 a $10 \mathrm{MW} / \mathrm{cm}^{2}$ quand le flux émis par un laser monochromatique de puissance $20 \mathrm{~mW}$ est concentré dans la tache de diffraction alors que pour les mêmes objectifs dont le champ est éclairé uniformément par une lampe à arc au mercure de forte luminance (40 000 stilbs), dont environ $2 \%$ de la puissance totale peut être estimée utilisée pour l'irradiation, les densités spatiales de puissance sont $10^{6}$ fois plus faibles. $\grave{A}$ rendement égal, il est donc possible d'envisager un effet identique pour des temps d'excitation réduits dans le même rapport.

Outre cette application très importante dans le secteur biomédical, la microscopie confocale permet l'étude d'une autre interaction lumière matière se traduisant par l'induction d'un courant électrique par irradiation lumineuse ou "OBIC" (Optical Beam Induced Current). Le balayage à l'aide d'un spot lumineux d'un matériau semi-conducteur par exemple permet d'y localiser les zones photosensibles par superposition de l'image passive de l'objet obtenue en lumière réfléchie et de son image active obtenue en captant le courant photoélectrique qu'il produit s'il est illuminé, l'analyse de ce signal permettant d'apprécier le rendement de cette transformation. De façon générale, séparer grâce à la faible profondeur de champ les défauts de volume de ceux de surface, repérer les dislocations sur un composant fini, contrôler leur microgéométrie dans trois directions sont des moyens répondant aux préoccupations du secteur de la microélectronique. Il en est de même en science de matériaux où cette microscopie est appliquée à l'étude de leur structure, à la détection des défauts et impuretés, à la caractérisation des états de surface et d'interface, au repérage des dislocations et joints de grain. La possibilité d'analyser de très fines tranches en microscopie confocale favorise l'étude des matériaux en couches minces. En lumière polarisée, ce qu'émet souvent naturellement un laser, on a accès à l'existence de biréfringences et activités, qu'elles soient naturelles ou provoquées sous l'action d'un champ électrique ou d'une induction magnétique (effets Pockels ou Faraday)

Notons encore que le microscope confocal à balayage laser utilisant une source de lumière cohèrente est tout à fait adapté à l'examen des objets de phase s'il lui est associé une méthode classiquement mise en cuvre comme le contraste de phase ou le contraste interférentiel. L'amélioration du contraste, la faible profondeur de champ, la diminution du bruit rendent possible avec un instrument confocal en réflexion l'examen d'objets typiquement transparents dont on peut mettre en évidence les différents interfaces (membranes biologiques par exemple).

\section{Références}

[01] Boutry G.A., Optique instrumentale (Masson, Paris, 1946).

[02] Bruhat G., Traité de polarimétrie (Éd. Revue d'Optique, Paris, 1930)

[03] Köhler A., "Ein neues Beleuchtungsverfahren für mikrophotographische Zwecke", $Z$. Wiss. Mikr. 67 (1893) 244

[04] Policard A., Bessis M. et Locquin M. Traité de microscopie (Masson, Paris, 1957).

[05] Terrien J., La microscopie (P.U.F., Paris, 1968).

[06] Locquin M. et Langeron M., Manuel de microscopie (Masson, Paris, 1978).

[07] Pluta M., Advanced light microscopy: Vol 1 : Principles and basic properties, Vol 2 Specialized method, Vol. 3 : Measuring techniques, (PWN-Polish Scientific Publishers, Warzawa \&Elsevier, Amsterdam, 1989).

[08] Roblin G., Microscopie optique, Dossier pédagogique $N^{\circ} 44$ (ADASTA, ClermontFerrand, 1995). 
[09] Zernicke F., "Das Phasenkontrastverfahren bei den mikroskopischen Beobachtung", $Z$. Physik 36 (1935) 848

[10] Bennett A.H., Osterberg H. Jupnik H. and Richards O.W., Phase microscopy (J.Wiley and sons, Londres, 1951).

[11] Lebedeff A.A., "L'interféromètre à polarisation et ses applications", Rev. Opt. 9 (1930) 385 .

[12] Françon M., Le microscope à contraste de phase et le microscope interférentiel (Éditions du CNRS, Paris, 1954).

[13] Françon M. et Mallick S., Polarization interferometers, (J.Wiley and sons, Londres, 1971).

[14] Germain G. et Roblin G., "Étude de la compensation d'un interféromètre à dédoublement latéral de type Jamin-Lebedeff", Nowv. Rev. Optique 6 (1975) 215-222.

[15] Weill A.R. et Nomarski G., "Application à la métallographie des méthodes interférentielles à deux ondes polarisées", Rev. Métallurgie 52 (1955) 121.

[16] Nomarski G. et Roblin G., "Localisation longitudinale des surfaces par interfèrométrie à modulation de phase". Nouv. Revue Opt. Appl. 2 (1971) 105-113.

[17] Roblin G., "La mesure des déphasages en microscopie par interférométrie à modulation de phase", J. Optics 8 (1977) 309-318.

[18] De Hoff R.T. et Rhines F.N., Microscopie quantitative (Masson, Paris, 1972).

[19] Roblin G., Bernstein L., Prévost M. et Slucki G., "Étude d'un microscope optique à balayage appliqué à la fluorimétrie", J. Optics 17 (1986) 259-269.

[20] Wilson T . and Sheppard C., Theory and practice of scanning optical microscopy (Academic Press, London, 1984)

[21] Sheppard C.J.R. and Choudhury A., Image formation in the scanning microscope, Optica Acta 24 (1977) 1051-1073.

[22] Wilke V., Gödecke U. und Seidel P., "Laser-Scan-Mikroskop", Laser und Optoelektronik (1983) 93-101.

[23] Dixon A.J., Doe N. and Pang T.-M., "Industrial applications of confocal scanning optical microscopy", Scanning Imaging Technology, SPIE 809 (1987), 36-43. 